Portland State University

PDXScholar

$1-1-2010$

\title{
Modeling Terahertz Diffuse Scattering from Granular Media Using Radiative Transfer Theory
}

Kyung Moon Nam

Portland State University

Follow this and additional works at: https://pdxscholar.library.pdx.edu/open_access_etds Let us know how access to this document benefits you.

\section{Recommended Citation}

Nam, Kyung Moon, "Modeling Terahertz Diffuse Scattering from Granular Media Using Radiative Transfer Theory" (2010). Dissertations and Theses. Paper 380.

https://doi.org/10.15760/etd.380

This Thesis is brought to you for free and open access. It has been accepted for inclusion in Dissertations and Theses by an authorized administrator of PDXScholar. Please contact us if we can make this document more accessible: pdxscholar@pdx.edu. 
Modeling Terahertz Diffuse Scattering from Granular Media

Using Radiative Transfer Theory

by

Kyung Moon Nam

A thesis submitted in partial fulfillment of the requirements for the degree of

Master of Science

in

Electrical and Computer Engineering

Thesis Committee:

Lisa M. Zurk, Chair

Donald Duncan

James McNames

Portland State University

(C)2011 


\begin{abstract}
Terahertz $(\mathrm{THz})$ spectroscopy can potentially be used to probe and characterize inhomogeneous materials, however spectroscopic identification of such materials from spectral features of diffuse returns is a relatively underdeveloped area of study. In this thesis, diffuse $\mathrm{THz}$ scattering from granular media is modeled by applying radiative transfer (RT) theory for the first time in $\mathrm{THz}$ sensing. Both classical RT theory and dense media radiative transfer (DMRT) theory based on the quasi-crystalline approximation (QCA) are used to calculate diffuse scattered intensity. The numerical solutions of the vector radiative transfer equations (VRTE) were coded and calculated in MATLAB.

The diffuse scattered field from compressed Polyethylene (PE) pellets containing steel spheres was measured in both transmission and reflection using a THz time domain spectroscopy (THz-TDS) system. Measurement results showed energy redistribution by granular media due to volume scattering as well as angle dependent spectral features due to Mie scattering. The RT model was validated by successfully reproducing qualitative features observed in experimental results.

Diffuse intensity from granular media containing Teflon, lactose sugar, and C4 explosive was then calculated using the RT models. Simulation results showed
\end{abstract}


the amplitude of diffuse intensity is affected by factors such as grain size, fractional volume of grains, thickness of scattering layer, and scattering angles. Spectral features were also observed in the diffuse intensity spectra from media containing grains with $\mathrm{THz}$ spectral signatures. The simulation results suggest the possibility of identifying materials from diffuse intensity spectra. 


\section{Acknowledgments}

I would like to thank my advisor Dr. Lisa Zurk for her guidance and support during this research. I would also like to thank Dr. Jian Chen for his assistance with the measurements, Jorge Quijano for his help in understanding radiative transfer theory, and Scott Schecklman, Gabe Kniffin, and Sam Henry for their helpful suggestions. I am also grateful to all my fellow students at the Northwest Electromagnetics and Acoustics Research Laboratory at Portland State University for their help and support during my research. In addition, I would like to thank Dr. Donald Duncan and Dr. James McNames for serving on my committee. Finally, I would like to acknowledge the National Science Foundation for funding this work. 


\section{Contents}

Abstract $\quad$ i

Acknowledgments

List of Tables vii

List of Figures viii

1 Introduction 1

1.1 Thesis Work ........................... 4

1.1.1 Contributions .................... 5

1.2 Thesis Summary ....................... 5

$\begin{array}{lll}2 & \text { Volume Scattering Models } & 7\end{array}$

2.1 Radiative Transfer Theory . . . . . . . . . . . . . . . 8

2.1.1 Vector Radiative Transfer Equation . . . . . . . . . . 9

2.1 .2 Reduced Intensity . . . . . . . . . . . . . . . 13

2.1 .3 Diffuse Intensity . . . . . . . . . . . . . 15

2.2 Dense Media Radiative Transfer Theory . . . . . . . . . . . . . 16 
2.2.1 Independent Scattering and Correlated Scattering . . . . . 17

2.2.2 Pair Distribution and Structure Factor . . . . . . . . 18

2.2.3 Quasi-Crystalline Approximation ............ 20

2.2.4 DMRT Equation and Its Solutions . . . . . . . . . . 24

2.3 Conservation of Energy . . . . . . . . . . . . . . . . 25

3 Terahertz Measurement and Results 28

3.1 Sample Preparation . . . . . . . . . . . . . . . . . 28

3.2 Measurement Process and Data Analysis . . . . . . . . . . . 29

3.2.1 Transmission Measurements . . . . . . . . . . 30

3.2.2 Reflection Measurements . . . . . . . . . . . . 33

3.3 Modeled Transmission and Reflection Response . . . . . . . . 35

3.4 Results and Discussion _. . . . . . . . . . . . . . . . 39

4 Simulation Results $\quad 44$

4.1 Simulations of Diffuse Specific Intensity . . . . . . . . . . . . . 44

4.1.1 Simulation Results for Medium 1 . . . . . . . . 46

4.1.2 Simulation Results for Medium 2 . . . . . . . . . 50

4.1.3 Simulation Results for Medium 3 . . . . . . . . . 53

4.2 Conclusion . . . . . . . . . . . . . . . . 56

5 Conclusions and Future Work $\quad 59$ 


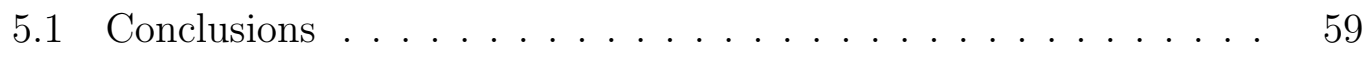

5.2 Future Work ......................... 61

$\begin{array}{ll}\text { References } & 63\end{array}$

$\begin{array}{ll}\text { Appendix A: Phase Matrix } & 70\end{array}$

Appendix B: Reflectivity Matrix and Transmissivity Matrix

Appendix C: Numerical Solution for Diffuse Intensity 


\section{List of Tables}

2.1 Four classes of random discrete particles from [33] . . . . . . . 19

3.1 Fractional volume of metal spheres, $f_{v}$, and the thickness, $d$, of two pellet samples . . . . . . . . . . . . . . . . . . 28

3.2 The samples used and the scattering angles observed for $\mathrm{THz}$

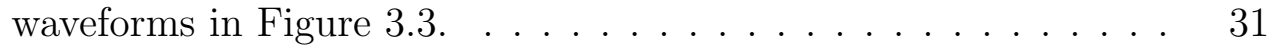

3.3 The samples used and the scattering angles observed for $\mathrm{THz}$ waveforms in Figure $3.6 . \ldots . \ldots . \ldots . \ldots 34$

3.4 Size parameter, albedo, and optical depth for samples . . . . . . 36

4.1 Parameters for granular media used in simulations. . . . . . . . . 46

4.2 Material properties of three granular media and their influence on diffuse intensity spectra . . . . . . . . . . . . . 58 


\section{List of Figures}

2.1 The incident intensity $I_{\text {in }}$ in the direction $(\hat{s})$ passes through a small volume $d V$ containing particles and is attenuated while the intensity $I$ from the direction $\left(\hat{s}^{\prime}\right)$ scatters intensity into the direction $(\hat{s})$. The transmitted intensity $I_{\text {out }}$ is the summation of the attenuated incident intensity from the $(\hat{s})$ direction and the scattered intensity from the $\left(\hat{s}^{\prime}\right)$ direction. . . . . . . . . 9

2.2 The geometrical configuration of a plane wave incident on a slab of a homogeneous medium containing spherical particles. . . . . .

2.3 Diagram of (a) the reflected intensity, $I_{r}$, reduced intensity, $I_{r i}$, and (b) diffuse intensity, $I_{d}$ observed from a slab containing particles illuminated by an obliquely incident intensity. . . . . . . . .

2.4 Extinction rate as a function of fractional volume for Teflon spheres (dashed line: independent scattering, solid line: QCA): $n_{s}=1.355$, $n_{b}=1.33, a=0.075 \mu \mathrm{m}, \lambda=0.6328 \mu \mathrm{m}, k a=0.99$. This plot is reproduced from $[32] \ldots \ldots \ldots \ldots \ldots$ 
2.5 (a) Pair distribution function and (b) structure factor for hard spheres with the diameter of $1 \mathrm{~mm} \ldots \ldots 20$

2.6 Conservation of power in classical RT model: (a) incident power flux $\left(F_{i}\right)$ and the summation of reflected power flux $\left(F_{r}\right)$, reduced power flux $\left(F_{r i}\right)$, and diffuse power flux $\left(F_{d}\right)$, and (b) (upper) reduced power flux and (lower) diffuse power flux for $f_{v}=5 \%, a$ $=50 \mu \mathrm{m}, d=5 \mathrm{~mm}, n_{0}=1, n_{1}=1.3, n_{2}=1, n_{p}=1.5$, and normal incidence $\left(\theta_{i}=0^{\circ}\right) \ldots \ldots \ldots \ldots$

2.7 Conservation of power in DMRT based on QCA model: (a) incident power flux $\left(F_{i}\right)$ and the summation of reflected power flux $\left(F_{r}\right)$, reduced power flux $\left(F_{r i}\right)$, and diffuse power flux $\left(F_{d}\right)$, and (b) (upper) reduced power flux and (lower) diffuse power flux for $f_{v}=20 \%, a=50 \mu \mathrm{m}, d=5 \mathrm{~mm}, n_{0}=1, n_{1}=1.3, n_{2}=1, n_{p}$ $=1.5$, and oblique incidence $\left(\theta_{i}=31.21^{\circ}\right) \ldots \ldots \ldots$

3.1 Compressed PE powder pellets containing steel spheres: sample 1 (left) and sample 2 (right). . . . . . . . . . .

3.2 Experiment setup for transmission measurement at a normal incidence, $\theta_{i}=0^{\circ}$ and $\theta_{s}=140^{\circ} \ldots \ldots \ldots \ldots$ 
3.3 The transmission measurement at normal incidence $\left(\theta_{i}=0^{\circ}\right)$ : (a) $\mathrm{THz}$ waveforms with no sample at $\theta_{s}=180^{\circ}$ and (b) no sample at $\theta_{s}=160^{\circ}$. (c) The transmitted waves through sample 2 at $\theta_{s}=180^{\circ}$. (d) - (g) The diffuse scattered waves from sample 2 at $\theta_{s}=160^{\circ}$ (different realizations). The curves, (a) and (c) were scaled by a factor of $0.015 \ldots \ldots \ldots \ldots \ldots \ldots \ldots$

3.4 The power spectral density of the waves (a) - (g) in Figure 3.3. . 32

3.5 Experiment setup for reflection measurement at an oblique incidence, $\theta_{i}=29^{\circ}$ and off-specular measurement, $\theta_{s}=-6^{\circ}$. . . . .

3.6 The reflection measurement at oblique incidence $\left(\theta_{i}=29^{\circ}\right)$ : (a) the specular reflection from a copper sheet $\left(\theta_{s}=29^{\circ}\right)$ and $(\mathrm{b})$ the off-specular angle $\left(\theta_{s}=-6^{\circ}\right)$ measurement from a copper sheet. (c) The reflected waves at the upper boundary (1) and at the lower boundary (2) of sample 1 . Curves (d) - (f) show the diffuse scattered waves for 3 of the 36 realizations from sample 1 at offspecular angle $\left(\theta_{s}=-6^{\circ}\right)$. The curve, (a) was scaled by a factor of 0.01 , and the curve, (c) was scaled by a factor of 0.015 . . . 
3.7 The power spectral density of the waves (a) - (f) from Figure 3.6. The curve (c-1) is the power spectral density from the first pulse and the curve (c-2) is that from the second pulse in time domain waveforms for sample $1 \ldots \ldots \ldots \ldots \ldots$

3.8 Results calculated from the RT model: (a) contrast between the diffuse specific intensity from metal spheres in sample 1 and from air voids $\left(f_{v}=20 \%, a=2 \mu \mathrm{m}\right)$ in transmission mode $\left(\theta_{i}=0^{\circ}\right.$ and $\left.\theta_{s}=139.7^{\circ}\right)$, and $(\mathrm{b})$ contrast between the diffuse specific intensity from the metal spheres in sample 1 and that from the air voids in reflection mode $\left(\theta_{i}=29^{\circ}\right.$ and $\left.\theta_{s}=-6.2^{\circ}\right) \ldots \ldots 38$

3.9 Comparison between the normalized intensity of measured data and simulated results in transmission mode: (a) at $\theta_{s}=180^{\circ}$, (b) at $\theta_{s}=160^{\circ},(\mathrm{c})$ at $\theta_{s}=140^{\circ}$, and $(\mathrm{d})$ at $\theta_{s}=120^{\circ} \ldots \ldots \ldots$ 
3.10 Comparison between the normalized intensity of measured data and simulated results in reflection mode: (a) (1st pulse) the reflected intensity bounced at the upper boundary of sample 1 and (2nd pulse) the summation of the reduced intensity and diffuse intensity at specular angle. The reduced intensity was bounced at the lower boundary once, but multiple times. The diffuse intensity is negligible. (b) The diffuse intensity at off-specular angle

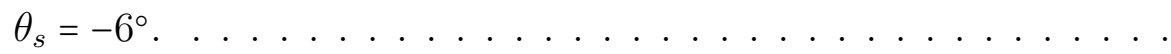

4.1 Geometrical configuration assumed in RT simulation. . . . . . . . 46

4.2 Diffuse specific intensity from medium 1 obtained by varying the size of air voids: $d=5 \mathrm{~mm}$ and $f_{v}=1 \%$. (black curve) The curve is proportional to $f^{4} \ldots \ldots \ldots \ldots \ldots$

4.3 Diffuse specific intensity from medium 1 obtained by varying the thickness $d$ of the medium: $a=38.8 \mu \mathrm{m}$ and $f_{v}=20 \% \ldots \ldots$.

4.4 Diffuse specific intensity as a function of scattering angle at 1 THz. As in Figure 4.3, $a=38.8 \mu \mathrm{m}$ and $f_{v}=20 \% \ldots \ldots \ldots$

4.5 Diffuse specific intensity from medium 1 obtained by varying the fractional volume of the air voids: $a=38.8 \mu \mathrm{m}$ and $d=5 \mathrm{~mm}$. .

4.6 Lactose permittivity: real part $\epsilon^{\prime}$ (upper curve) and imaginary part $\epsilon^{\prime \prime}$ (lower curve) $[37,38] . \ldots \ldots \ldots \ldots$ 
4.7 The diffuse specific intensities in reflection mode for medium 2 in

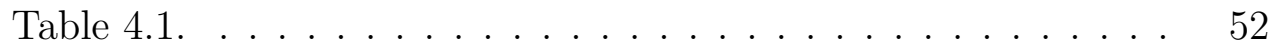

4.8 The diffuse specific intensities in transmission mode for medium

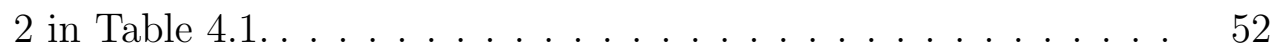

4.9 C4 permittivity: real part $\epsilon^{\prime}$ (upper curve) and imaginary part $\epsilon^{\prime \prime}$ (lower curve) $[39] . \ldots \ldots \ldots \ldots$. . . . . . . . . . . . . . . . . 53

4.10 The diffuse specific intensities in reflection mode for medium 3 in Table $4.1 . \ldots \ldots \ldots \ldots \ldots \ldots \ldots$

4.11 The diffuse specific intensities in transmission mode for medium 3 in Table $4.1 \ldots \ldots \ldots \ldots \ldots \ldots$ 


\section{Chapter 1}

\section{Introduction}

The terahertz $(\mathrm{THz})$ frequency band lies between the neighboring microwave and mid-infrared bands in the electromagnetic (EM) spectrum. While the microwave and optical regions of the spectrum have been thoroughly utilized, the $\mathrm{THz}$ band has only recently been explored owing to recent advances in ultrafast optoelectronics and micro-scale semiconductors which have allowed the development of sufficiently efficient $\mathrm{THz}$ sources and sensitive $\mathrm{THz}$ detectors $[1,2]$. THz radiation has shown great potential for a variety of applications such as medical imaging, security screening, and communications $[3,4,5,6,7,8]$.

THz spectroscopy has been shown to be useful for probing and characterizing inhomogeneous materials (such as concealed explosives and illegal drugs, layered media, etc.) $[5,6]$. Many materials of interest (i.e. explosives and chemical and biological substances) have characteristic spectral features (fingerprints) in $\mathrm{THz}$ frequency region, enabling the identification of such materials. $\mathrm{THz}$ waves also penetrate through non-polar materials such as clothing and packaging. In addition, THz radiation is nonionizing and is safe to use on live biological tissues at low power levels. This combination of properties makes $\mathrm{THz}$ attractive for detection of concealed drugs and/or explosives in the mail system or in airport 
security screening.

Recent work suggests scattering may affect the spectral returns from target materials in real-world scenarios $[9,10,11,12,13]$. The effect of rough surface scattering on $\mathrm{THz}$ reflection spectra from explosive materials has been investigated using the Kirchhoff approximation (KA) and small perturbation method (SPM) models [12]. Diffuse scattering from lactose samples $[11,13]$ and some explosives and related compounds (ERCs) with rough surfaces have also been measured [9]. In addition to rough surface scattering, volume scattering from granular inhomogeneities is likely to influence the spectral returns.

For example, commonly used plastic explosives contain explosive crystals and air voids in a plastic filler. The crystals and air voids are on the order of hundreds of microns in size - the same scale as $\mathrm{THz}$ wavelengths. This is likely to result in a significant amount of volume scattering, which will have a frequency dependent effect on the spectral returns.

To date, there has been relatively little investigation into $\mathrm{THz}$ scattering from granular media. THz scattering from granular materials has been studied using dense media theory and $\mathrm{THz}$ transmission measurements [10]. In addition, the extinction coefficient of different sized grains have been measured in transmission mode and compared to Mie theory predictions [14]. The propagation of coherent $\mathrm{THz}$ waves in a random medium consisting of Teflon spheres has also 
been measured as a function of the length of the medium and the scattering mean free path has been extracted [15]. Finally, THz propagation in strongly scattering random dielectric media has been investigated as well, using a Monte Carlo model and transmission measurements [16]. All these works explored attenuation of coherent energy due to scattering effects in granular random media from transmission measurements.

In contrast, this thesis focuses on the modeling and measurement of diffuse scattering from volume scattering. When EM waves propagate through a discrete random medium, scattering occurs due to inhomogeneities, resulting in a decrease in the coherent field and a corresponding increase in the incoherent field. The incoherent field also increases with optical depth and albedo. In extreme cases, the coherent returns may not be strong enough to provide any useful information about the medium's material properties, necessitating the use of the incoherent, diffuse returns instead. Also, the scattering angle-dependent diffuse volume scattering may influence the scattering angle dependent diffuse rough surface scattering.

Volume scattering in random media has been investigated extensively in microwave and optical ranges. Volume scattering of $\mathrm{THz}$ waves, however, has not received the same attention due to high absorption and the longer wavelengths [17]. Recently the diffuse scattering of $\mathrm{THz}$ radiation has been measured 
using Teflon grains [18], and has since become an important concern [17]. The study of diffuse volume scattering of $\mathrm{THz}$ radiation is therefore in its infancy. Also, the identification of spectral features from the diffuse scattering in homogeneous materials has not yet been considered.

\subsection{Thesis Work}

The focus of this thesis is modeling the $\mathrm{THz}$ scattering in granular media using radiative transfer (RT) theory and validating the RT model with measurements. Knowledge of $\mathrm{THz}$ volume scattering effects on measured spectra will help researchers develop spectroscopic identification and/or classification algorithms for future $\mathrm{THz}$ detection systems. Both classical radiative transfer (RT) theory for independent scattering and modified radiative transfer for dense media are used to model these diffuse scattering effects. This work constitutes the first time RT theory has been applied to $\mathrm{THz}$ spectroscopic sensing.

Numerical solutions of the radiative transfer equation (RTE) are derived for the case of random media with plane-parallel boundaries as is commonly done in the literature. The solutions are then implemented numerically in MATLAB with various parameters.

Samples consisting of $1 \mathrm{~mm}$ diameter steel spheres embedded in polyethylene (PE) were prepared and measured in both transmission and reflection modes 
to validate theoretical predictions. The transmitted diffuse field resulting from illuminating the samples with normally incident $\mathrm{THz}$ radiation was measured at various scattering angles using a $\mathrm{THz}$ time domain spectroscopy (THz-TDS) system. The reflected diffuse field at oblique incidence is also measured near the backscattering angle.

\subsubsection{Contributions}

- Implemented numerical solutions of the radiative transfer equation in MATLAB

- Validated predictions of RT model by comparison to measurement results from multiple samples with different volume inclusions

- Explored diffuse volume scattering from granular media containing Teflon, C4 explosive, and lactose sugar using RT models

\subsection{Thesis Summary}

Chapter 2 explores the propagation and scattering of electromagnetic waves in a medium containing spherical particles, and its mathematical description through classical radiative transfer theory and dense media radiative transfer theory.

Chapter 3 presents the experimental results with samples containing steel spheres obtained using a THz-TDS system before comparing the experimental 
data and simulation results.

Chapter 4 compares simulation results from RT models using Teflon, which is almost transparent, to results using lactose and $\mathrm{C} 4$, which have spectral signatures in $\mathrm{THz}$.

Chapter 5 summarizes this thesis work and describes the future work. 


\section{Chapter 2}

\section{Volume Scattering Models}

Analytical wave theory and radiative transfer (RT) theory are two methods used to solve scattering problems in discrete random media. The application of analytical theory to real-world problems may be complicated due to the fact that it is based on a rigorous solution to electromagnetic wave theory, including the multiple scattering, interference effects, and diffraction. On the other hand, RT theory is simple and practical as it uses the addition of power instead of fields and assumes independent scattering [19].

Dense media radiative transfer (DMRT) theory takes into account correlated scattering effects and has the same form as the radiative transfer equation. DMRT is useful in dense media, where the correlation between particles cannot be ignored [20]. In this chapter, the RT theory and DMRT will be used to study the diffuse scattering from a medium containing randomly distributed particles.

Section 2.1 will introduce the classical RT theory. The basic concepts of transport of intensity in a medium containing scatterers are presented, the vector radiative transfer equation (VRTE) for the plane-parallel problem is then introduced, followed by the solutions of the VRTE. Section 2.2 describes the DMRT theory based on the quasi-crystalline approximation (QCA). 


\subsection{Radiative Transfer Theory}

Radiative transfer theory was originally used to study stellar radiation in astrophysics [21], but its use has since spread to other fields. One main application is active and passive microwave remote sensing of terrain, particularly for plane parallel geometries $[22,23]$.

RT theory is derived from the transport of energy (or intensity) and it assumes independent scattering. An important quantity in RT theory is the specific intensity, which is defined as the average power flux density within a unit frequency within a unit solid angle, and its unit is $\left[W m^{-2} s r^{-1} H z^{-1}\right](s r=$ steradian) [19]. When an electromagnetic wave passes through a random medium in a certain direction containing many particles whose refractive index is different from that of the background medium, the incident wave is attenuated due to both absorption and scattering in the medium, but received power increases due to redistribution of the scattered wave from other directions into the direction of observation as shown in Figure 2.1. In RT theory, the specific intensity quantifies this loss or gain in the incident wave in terms of the extinction coefficient, which represents loss of the specific intensity, and the phase matrix, which represents gain in the specific intensity. 


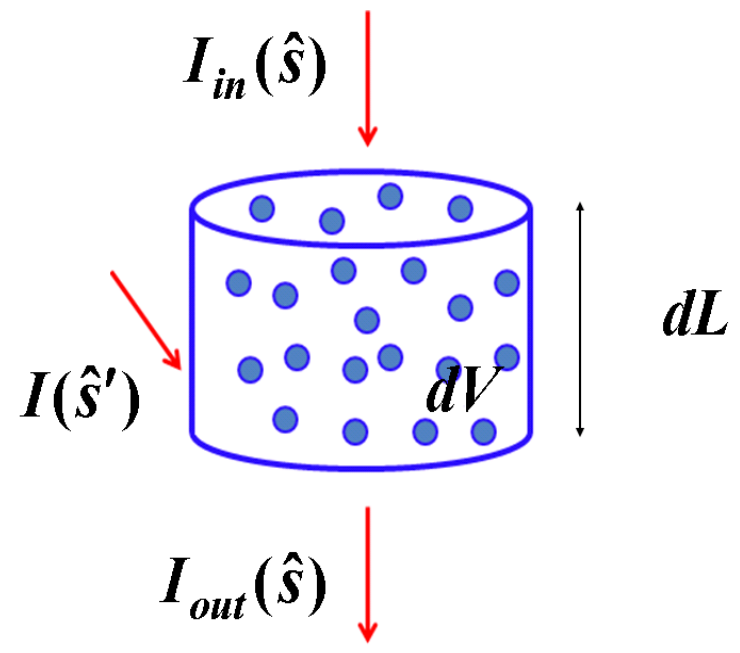

Figure 2.1: The incident intensity $I_{\text {in }}$ in the direction $(\hat{s})$ passes through a small volume $d V$ containing particles and is attenuated while the intensity $I$ from the direction $\left(\hat{s}^{\prime}\right)$ scatters intensity into the direction $(\hat{s})$. The transmitted intensity $I_{\text {out }}$ is the summation of the attenuated incident intensity from the $(\hat{s})$ direction and the scattered intensity from the $\left(\hat{s}^{\prime}\right)$ direction.

\subsubsection{Vector Radiative Transfer Equation}

The vector radiative transfer equation (VRTE) for a plane-parallel medium is used to model the diffuse intensity for discrete random media illuminated by a terahertz source. Consider an incident electromagnetic plane wave impinging obliquely on a plane-parallel slab containing spherical particles as shown in Figure 2.2. In this thesis, particles or grains will be assumed as spheres. The differential change in specific intensity with distance along the direction of propagation is expressed in the form of the VRTE as follows [22]: 


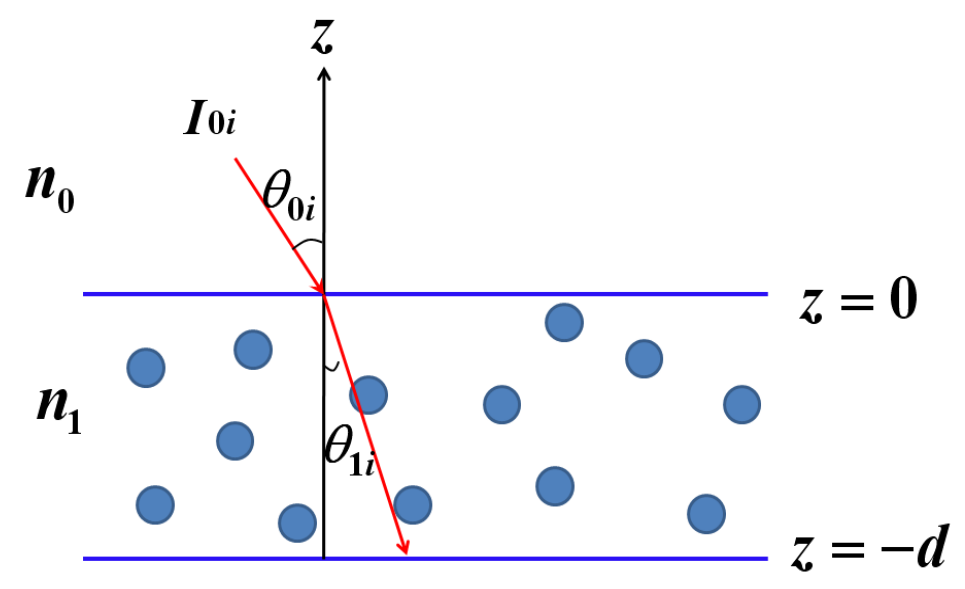

\section{$\boldsymbol{n}_{2}$}

Figure 2.2: The geometrical configuration of a plane wave incident on a slab of a homogeneous medium containing spherical particles.

$\cos \theta \frac{\mathrm{d}}{\mathrm{d} z} \bar{I}(\theta, \phi, z)=-\kappa_{e} \bar{I}(\theta, \phi, z)+\int_{0}^{2 \pi} \mathrm{d} \phi^{\prime} \int_{0}^{\pi} \mathrm{d} \theta^{\prime} \sin \theta^{\prime} \overline{\bar{P}}\left(\theta, \phi ; \theta^{\prime}, \phi^{\prime}\right) \bar{I}\left(\theta^{\prime}, \phi^{\prime}, z\right)$.

In $(2.1), \bar{I}(\theta, \phi, z)$ is the specific intensity, which is a vector of four components representing the four Stokes parameters, $I_{v}, I_{h}, U$, and $V$. The Stokes parameters are given by [24]

$$
\begin{aligned}
& I_{v}=\frac{\left|E_{v}\right|^{2}}{\eta}, \quad I_{h}=\frac{\left|E_{h}\right|^{2}}{\eta}, \\
& U=\frac{2}{\eta} \operatorname{Re}\left(E_{v} E_{h}^{*}\right), \quad V=\frac{2}{\eta} \operatorname{Im}\left(E_{v} E_{h}^{*}\right),
\end{aligned}
$$

where $\eta=\sqrt{\frac{\mu}{\epsilon}}$ is the wave impedance. In $(2.1), \kappa_{e}$ is called extinction coefficient, which is the summation of the scattering coefficient $\left(\kappa_{s}\right)$ and absorption coefficient $\left(\kappa_{a}\right), \kappa_{e}=\kappa_{s}+\kappa_{a}$. Because independent scattering is assumed, the 
scattering coefficient can be written as $\kappa_{s}=n_{o} \sigma_{s}$, where $n_{o}$ is the number of particles per unit volume and $\sigma_{s}$ is the scattering cross section for a particle. The absorption coefficient is divided into absorption in the particles and in the background medium, $\kappa_{a}=\kappa_{a p}+\kappa_{a b}$. The absorption in the particles can be written as $\kappa_{a p}=n_{o} \sigma_{a}$, where $\sigma_{a}$ is the absorption cross section for a particle. The scattering cross section and the absorption cross section for a single particle, $\sigma_{s}$ and $\sigma_{a}$ are calculated based on Lorenz-Mie theory $[24,25,26]$.

$$
\begin{aligned}
& \sigma_{s}=\frac{2 \pi}{k^{2}} \sum_{n=1}^{\infty}(2 n+1)\left\{\left|a_{n}\right|^{2}+\left|b_{n}\right|^{2}\right\}, \\
& \sigma_{t}=\frac{2 \pi}{k^{2}} \sum_{n=1}^{\infty}(2 n+1)\left\{\operatorname{Re}\left(a_{n}+b_{n}\right)\right\}, \\
& \sigma_{a}=\sigma_{t}-\sigma_{s},
\end{aligned}
$$

where $a_{n}$ and $b_{n}$ are scattering coefficients for a sphere. The absorption in the background medium can be written as $\kappa_{a b}=2 k_{b}^{\prime \prime}\left(1-f_{v}\right)$, where $k_{b}^{\prime \prime}$ is the imaginary part of the propagation constant of the background medium and $f_{v}$ is the fractional volume of the particles in the particle-background mixture. $\overline{\bar{P}}\left(\theta, \phi ; \theta^{\prime}, \phi^{\prime}\right)$ is a $4 \times 4$ phase matrix, which represents the link between the scattered intensity into the direction $(\theta, \phi)$ from the incident intensity in the direction $\left(\theta^{\prime}, \phi^{\prime}\right)$ (see Appendix A), where $0<\theta<\pi$ and $0<\phi<2 \pi$.

Initial conditions and boundary conditions are required to solve Equation (2.1). 
The incident wave in region 0 is given by

$$
\bar{I}_{0 i}\left(\pi-\theta_{0}, \phi_{0}, z=0\right)=\bar{F}_{o} \delta\left(\cos \theta_{0}-\cos \theta_{0 i}\right) \delta\left(\phi_{0}-\phi_{0 i}\right)
$$

where $\bar{F}_{o}$ is the amplitude vector of the incident power flux in the direction $\left(\theta_{0 i}, \phi_{0 i}\right)$. The boundary conditions are given as

$$
\begin{aligned}
\bar{I}^{-}(\pi-\theta, \phi, z=0) & =\overline{\bar{R}}_{10}(\theta) \bar{I}^{+}(\theta, \phi, z=0)+\overline{\bar{T}}_{01}\left(\theta_{o}\right) \bar{I}_{0 i}\left(\pi-\theta_{0}, \phi_{0}, z=0\right) \\
\bar{I}^{+}(\theta, \phi, z=-d) & =\overline{\bar{R}}_{12}(\theta) \bar{I}^{-}(\pi-\theta, \phi, z=-d)
\end{aligned}
$$

where $0<\theta<\pi / 2$, and intensities are divided into upward intensity $\bar{I}^{+}$and downward intensity $\bar{I}^{-}$. The reflectivity matrix $\overline{\bar{R}}_{a b}$ and the transmissivity matrix $\overline{\bar{T}}_{a b}$ express the relations between the incident and reflected Stokes vectors and between the incident and transmitted Stokes vectors, respectively, at a planar interface between medium $a$ and medium $b(a, b=0,1,2)$ with the refractive indices of $n_{a}$ and $n_{b}$, respectively [24]. The subscript $a$ represents the first medium and the subscript $b$ represents the second medium. The reflectivity matrix and the transmissivity matrix are presented in detail in Appendix B. 


\subsubsection{Reduced Intensity}

The specific intensity can be separated into the reduced intensity and the diffuse intensity [19]. The reduced intensity represents the coherent intensity as shown in Figure 2.3(a). Mathematically, it is the zeroth-order solution of Equation (2.1), which is calculated by setting $\overline{\bar{P}}=0$. This results in

$$
\cos \theta \frac{\mathrm{d}}{\mathrm{d} z} \bar{I}_{r i}(\theta, \phi, z)=-\kappa_{e} \bar{I}_{r i}(\theta, \phi, z)
$$

which, with boundary conditions from (2.5), gives the reduced intensity in closed form;

$$
\begin{aligned}
\bar{I}_{r i}^{+}(\theta, \phi, z) & =\exp \left[-\kappa_{e}(z+2 d) \sec \theta\right] \overline{\bar{F}}(\theta) \overline{\bar{R}}_{12}(\theta) \overline{\bar{T}}_{01}\left(\theta_{o}\right) \bar{I}_{o i}\left(\pi-\theta_{o}, \phi_{o}\right), \\
\bar{I}_{r i}^{-}(\pi-\theta, \phi, z) & =\exp \left(\kappa_{e} z \sec \theta\right) \overline{\bar{F}}(\theta) \overline{\bar{T}}_{01}\left(\theta_{o}\right) \bar{I}_{o i}\left(\pi-\theta_{o}, \phi_{o}\right),
\end{aligned}
$$

where $\overline{\bar{F}}=\left[\overline{\bar{U}}-\overline{\bar{R}}_{10}(\theta) \overline{\bar{R}}_{12}(\theta) \exp \left(-2 \kappa_{e} d \sec \theta\right)\right]^{-1}$ is a matrix that characterizes the effect of the dielectric slab [27]. Thus this solution assumes infinite reflections from the upper and the lower boundaries.

As shown in Figure 2.3(a), some amount of incident intensity is reflected at the upper boundary, resulting in reflected intensity $\left(I_{r}\right)$. The rest enters the second medium, which contains particles, and is attenuated as it passes through the medium, resulting in reduced intensity $\left(I_{r i}\right)$. The upward going reduced 
intensity in region 0 is given by $\bar{I}_{r i, 0}^{+}=\overline{\bar{T}}_{10} \bar{I}_{r i}^{+}$, while the downward going reduced intensity in region 2 is given by $\bar{I}_{r i, 2}^{-}=\overline{\bar{T}}_{12} \bar{I}_{r i}^{-}$.

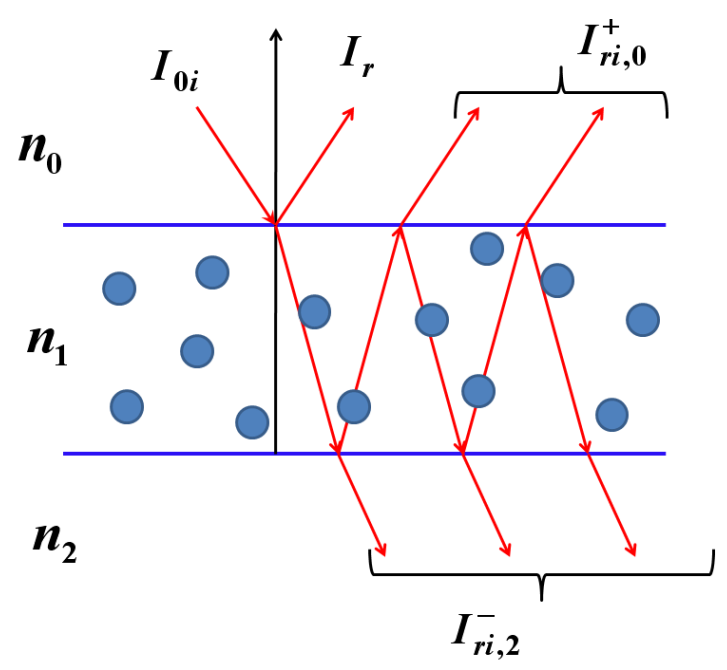

(a) Reduced Intensity

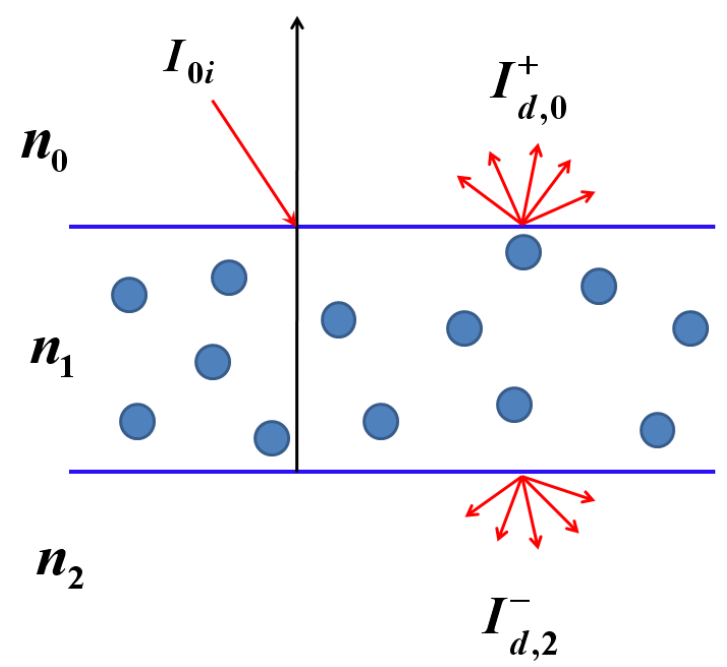

(b) Diffuse Intensity

Figure 2.3: Diagram of (a) the reflected intensity, $I_{r}$, reduced intensity, $I_{r i}$, and (b) diffuse intensity, $I_{d}$ observed from a slab containing particles illuminated by an obliquely incident intensity. 


\subsubsection{Diffuse Intensity}

The diffuse intensity represents the incoherent intensity traveling in all directions as shown in Figure 2.3(b). The upward going diffuse intensity in region 0 is

$\bar{I}_{d, 0}^{+}=\overline{\bar{T}}_{10} \bar{I}_{d}^{+}$and the downward going diffuse intensity in region 2 is $\bar{I}_{d, 2}^{-}=\overline{\bar{T}}_{12} \bar{I}_{d}^{-}$. The equation for the diffuse intensity is obtained by substituting $I=I_{r i}+I_{d}$ into Equation (2.1) and then subtracting Equation (2.6). The radiative transfer equation for diffuse intensity is given by

$$
\begin{aligned}
\cos \theta \frac{\mathrm{d}}{\mathrm{d} z} \bar{I}_{d}(\theta, \phi, z)= & -\kappa_{e} \bar{I}_{d}(\theta, \phi, z)+\int_{0}^{2 \pi} \mathrm{d} \phi^{\prime} \int_{0}^{\pi} \mathrm{d} \theta^{\prime} \sin \theta^{\prime} \overline{\bar{P}}\left(\theta, \phi ; \theta^{\prime}, \phi^{\prime}\right) \bar{I}_{d}\left(\theta^{\prime}, \phi^{\prime}, z\right) \\
& +\overline{\bar{P}}\left(\theta, \phi ; \theta_{1 i}, \phi_{1 i}\right) \bar{J}_{1}\left(\theta_{1 i}, \phi_{1 i}\right) \exp \left[-\kappa_{e}(z+2 d) \sec \theta_{1 i}\right] \\
& +\overline{\bar{P}}\left(\theta, \phi ; \pi-\theta_{1 i}, \phi_{1 i}\right) \bar{J}_{2}\left(\theta_{1 i}, \phi_{1 i}\right) \exp \left(\kappa_{e} z \sec \theta_{1 i}\right)
\end{aligned}
$$

where $\theta_{1 i}$ is the transmitted angle associated with the incident angle $\theta_{0 i}$ from medium 0 to medium 1 (obtained using the Snell's law), and $\bar{J}_{1}$ and $\bar{J}_{2}$ are given by

$$
\begin{aligned}
\bar{J}_{1}\left(\theta_{1 i}, \phi_{1 i}\right) & =\overline{\bar{F}}\left(\theta_{1 i}\right) \overline{\bar{R}}_{12}\left(\theta_{1 i}\right) \overline{\bar{T}}_{01}\left(\theta_{0 i}\right) \bar{I}_{0 i}\left(\pi-\theta_{0}, \phi_{0}\right) \\
& =\frac{n_{0}^{2} \cos \theta_{0 i}}{n_{1}^{2} \cos \theta_{1 i}} \overline{\bar{F}}\left(\theta_{1 i}\right) \overline{\bar{R}}_{12}\left(\theta_{1 i}\right) \overline{\bar{T}}_{01}\left(\theta_{0 i}\right) \bar{I}_{1 i}\left(\pi-\theta_{1}, \phi_{1}\right) \\
\bar{J}_{2}\left(\theta_{1 i}, \phi_{1 i}\right) & =\overline{\bar{F}}\left(\theta_{1 i}\right) \overline{\bar{T}}_{01}\left(\theta_{0 i}\right) \bar{I}_{0 i}\left(\pi-\theta_{0}, \phi_{0}\right) \\
& =\frac{n_{0}^{2} \cos \theta_{0 i}}{n_{1}^{2} \cos \theta_{1 i}} \overline{\bar{F}}\left(\theta_{1 i}\right) \overline{\bar{T}}_{01}\left(\theta_{0 i}\right) \bar{I}_{1 i}\left(\pi-\theta_{1}, \phi_{1}\right)
\end{aligned}
$$


where $\bar{I}_{1 i}\left(\pi-\theta_{1}, \phi_{1}\right)=\bar{F}_{o} \delta\left(\cos \theta_{1}-\cos \theta_{1 i}\right) \delta\left(\phi_{1}-\phi_{1 i}\right)$ and $\theta_{1}$ is the transmitted angle $\theta_{0}$ and $\phi_{1}=\phi_{o}$.

The third and the fourth terms of the right-hand side in Equation (2.8) represent the source of diffuse intensity from the incident intensity of a single scattering event. The single scattering solution can be calculated by removing the integral term which accounts for multiple scattering. Shin [22] calculated the first and second order solutions for Rayleigh particles by using an iterative method. Higher order solutions cannot be obtained analytically, so numerical methods are utilized to obtain the complete solution. Equation (2.8) is solved with boundary conditions for diffuse intensity,

$$
\begin{aligned}
\bar{I}_{d}^{-}(\pi-\theta, \phi, z=0) & =\overline{\bar{R}}_{10}(\theta) \bar{I}_{d}^{+}(\theta, \phi, z=0) \\
\bar{I}_{d}^{+}(\theta, \phi, z=-d) & =\overline{\bar{R}}_{12}(\theta) \bar{I}_{d}^{-}(\pi-\theta, \phi, z=-d) .
\end{aligned}
$$

The numerical solution for diffuse intensity is explained briefly in Appendix C.

\subsection{Dense Media Radiative Transfer Theory}

Radiative transfer theory is applicable to practical problems involving multiple scattering because of its mathematical simplicity. However, using an independent scattering assumption limits its use to low fractional volume of particles. As the fractional volume of particles increases, the scattering will be influenced by 
the coherent wave interaction among the particles. The dense media radiative transfer (DMRT) theory has been developed for the propagation and scattering of waves in dense media and applied to the microwave remote sensing of snow [28, 29]. In contrast to the classical radiative transfer theory, DMRT does not start with the transport of energy but is derived from electromagnetic wave theory based on the quasicrystalline approximation, with coherent potential (QCA-CP) for small particles and quasicrystalline approximation (QCA) for moderate size particles [20, 27, 28, 30, 31]. However, the DMRT retains the form of the classical radiative transfer equation, so the numerical solution for diffuse intensity can be calculated in the same method [20].

\subsubsection{Independent Scattering and Correlated Scattering}

Figure 2.4 illustrates the comparison between independent scattering and QCA in a medium containing discrete scatterers [32]. For the independent scattering, the extinction rate is linearly proportional to the fractional volume, which is not physical as fractional volume increases. For instance, when particles occupy the whole volume, scattering does not occur because the medium becomes homogeneous [33].

Table 2.1 shows four classes of random discrete scatterers. In dense media, the particle positions are correlated. Thus the imaginary part of the effective propagation constant $\left(K_{i}\right)$, which is the attenuation rate due to scattering and 


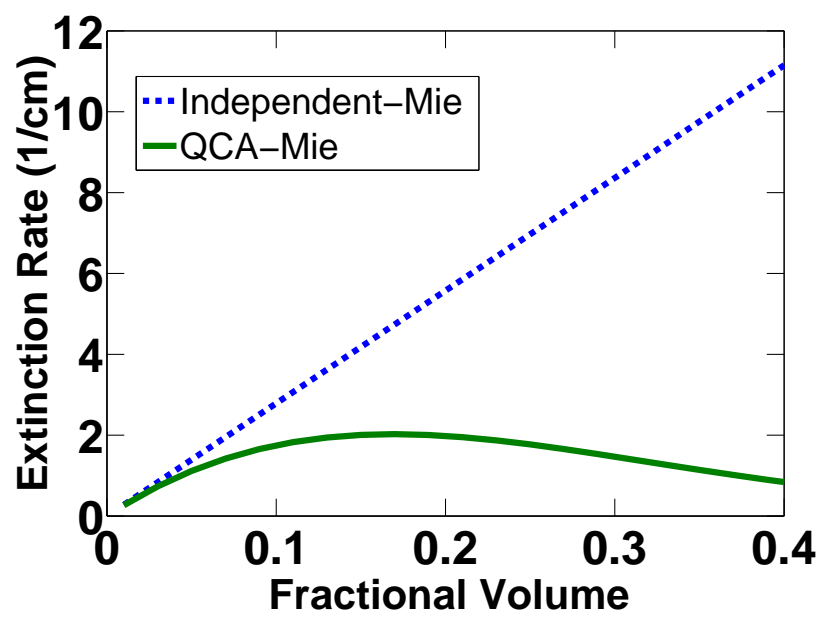

Figure 2.4: Extinction rate as a function of fractional volume for Teflon spheres (dashed line: independent scattering, solid line: QCA): $n_{s}=1.355, n_{b}=1.33$, $a=0.075 \mu \mathrm{m}, \lambda=0.6328 \mu \mathrm{m}, k a=0.99$. This plot is reproduced from [32].

absorption, is not linearly proportional to the fractional volume of particles. Especially in non-tenuous media, the real part of the effective propagation constant of the scatterers $\left(K_{r}\right)$ are considerably different from that of the background medium [33].

These facts show that as the fractional volume of particles increases, independent scattering is not appropriate and correlated scattering must be considered.

\subsubsection{Pair Distribution and Structure Factor}

In this thesis, the DMRT based on QCA from [29] is used to model high fractional volume granular media. Most of procedures follow the reference except a pair distribution function. A pair distribution function is related to a joint probability distribution of two particles' positions [33]. While the pair distribution 
Table 2.1: Four classes of random discrete particles from [33]

\begin{tabular}{|l||l|l|l|l|l|}
\hline Class & $\begin{array}{l}\text { Dielectric } \\
\text { property } \\
\text { of particles }\end{array}$ & $\begin{array}{l}\text { Particle } \\
\text { concentration }\end{array}$ & $\begin{array}{l}\text { Particle } \\
\text { positions }\end{array}$ & $K_{r}$ & $\begin{array}{l}\text { Relation } \\
\text { of } K_{i} \\
\text { with } f_{v}\end{array}$ \\
\hline \hline $\mathrm{A}$ & tenuous & sparse & independent & $\cong k_{b}$ & linear \\
\hline $\mathrm{B}$ & non-tenuous & sparse & independent & $\cong k_{b}$ & linear \\
\hline $\mathrm{C}$ & tenuous & dense & correlated & $\cong k_{b}$ & nonlinear \\
\hline $\mathrm{D}$ & non-tenuous & dense & correlated & $\begin{array}{l}\text { significantly } \\
\text { different } \\
\text { from } k_{b}\end{array}$ & nonlinear \\
\hline
\end{tabular}

function for adhesive particles was used in the reference, the pair distribution function for impenetrable spheres is used in this thesis. The Percus-Yevick (PY) approximation is used for the pair distribution function for impenetrable spheres to present the correlations of particle positions. The PY approximation is the closed form solution of the Ornstein-Zernike equation. Details are in [33, 34].

The positions of particles in a medium affect the scattering of electromagnetic waves [33]. As shown in Figure 2.5(a), when the particles are located far away from each other, the pair distribution function is close to unity and independent scattering occurs. However, when the particles approach each other, the pair distribution function fluctuates and two particles are not independent. As the fractional volume increases, two particles are less independent. The Fourier transform of the pair distribution is the structure factor, which is proportional to the phase matrix. As depicted in Figure 2.5(b), the structure factor fluctuates 
(a)

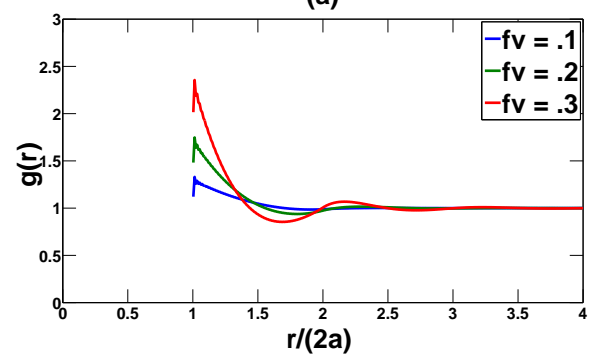

(b)

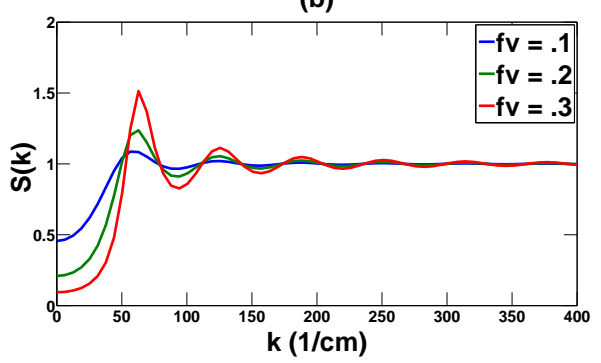

Figure 2.5: (a) Pair distribution function and (b) structure factor for hard spheres with the diameter of $1 \mathrm{~mm}$

more as the fractional volume of particles increases.

\subsubsection{Quasi-Crystalline Approximation}

This section presents a brief overview of DMRT based on QCA. Details can be found in $[20,29]$. For an incident plane wave impinging on a medium consisting of $N$ identical spherical particles, the field exciting the $l$ th particle is the sum of the incident wave and the scattered wave from all the other particles $j(j \neq l)$. Using the Foldy-Lax multiple scattering equation in matrix form, the exciting field is given as [20],

$$
\bar{w}^{(l)}=\sum_{\substack{j=1 \\ j \neq l}}^{N} \overline{\bar{\sigma}}\left(k \bar{r}_{l} r_{j}\right) \overline{\bar{T}}^{(j)} \bar{w}^{(j)}+e^{i \bar{k}_{i} \cdot \bar{r}_{l}} \bar{a}_{i n c}
$$

where $\overline{\bar{\sigma}}\left(k \overline{r_{l} r_{j}}\right)$ is a matrix that represents vector translation formula from the center of the $l$ th particle to the center of the $j$ th particle, $\bar{a}_{i n c}$ is a vector representing the coefficient of the incident wave when expanded into spherical waves, 
and $\overline{\bar{T}}$ is the T-matrix for the particles. The T-matrix is used to describe the linear relationship between the scattered field coefficients and the exciting field coefficients, and the T-matrix for spherical particles is defined as

$$
\overline{\bar{T}}=\left[\begin{array}{cc}
T_{n}^{(M)} & 0 \\
0 & T_{n}^{(N)}
\end{array}\right] .
$$

The T-matrix elements are given [24]

$$
\begin{aligned}
T_{n}^{(N)} & =-\frac{k_{p}^{2} a^{2} j_{n}\left(k_{p} a\right)\left[k a j_{n}(k a)\right]^{\prime}-k^{2} a^{2} j_{n}(k a)\left[k_{p} a j_{n}\left(k_{p} a\right)\right]^{\prime}}{k_{p}^{2} a^{2} j_{n}\left(k_{p} a\right)\left[k a h_{n}(k a)\right]^{\prime}-k^{2} a^{2} h_{n}(k a)\left[k_{p} a j_{n}\left(k_{p} a\right)\right]^{\prime}} \\
T_{n}^{(M)} & =-\frac{j_{n}\left(k_{p} a\right)\left[k a j_{n}(k a)\right]^{\prime}-j_{n}(k a)\left[k_{p} a j_{n}\left(k_{p} a\right)\right]^{\prime}}{j_{n}\left(k_{p} a\right)\left[k a h_{n}(k a)\right]^{\prime}-h_{n}(k a)\left[k_{p} a j_{n}\left(k_{p} a\right)\right]^{\prime}}
\end{aligned}
$$

where $j_{n}$ is the spherical Bessel function and $h_{n}$ is the spherical Hankel function. The subscript $p$ denotes a particle, $k_{p}$ is the propagation constant inside of the particle, and $k$ is the propagation constant in the background medium.

Equation (2.11) is calculated using conditional averaging, and then the averaging exciting field under QCA is given as [20]

$$
\bar{w}\left(\bar{r}_{l}\right)=n_{o} \int_{z_{j}>0} d \bar{r}_{j} g\left(\bar{r}_{j}-\bar{r}_{l}\right) \overline{\bar{\sigma}}\left(k \overline{r_{l} r_{j}}\right) \overline{\bar{T}} \bar{w}\left(\bar{r}_{j}\right)+e^{i \bar{k}_{i} \cdot \bar{r}_{l}} \bar{a}_{i n c}
$$

where $n_{o}$ is the number of spheres per volume, $g\left(\bar{r}_{j}-\bar{r}_{l}\right)$ is the pair distribution function, and $\bar{w}\left(\bar{r}_{l}\right)$ is the conditional average of the exciting field of particles 
at $\bar{r}_{l}$.

The above equation is expanded into vector spherical waves, which are a homogeneous system of equations known as the Lorentz-Lorenz (L-L) law [20]:

$$
\begin{aligned}
X_{\nu}^{(M)}= & -2 \pi n_{o} \sum_{n=1}^{N_{\max }} \sum_{p=|n-\nu|}^{|n+v|}(2 n+1)\left[L_{p}(k, K / b)+M_{p}(k, K / b)\right] \\
\times & {\left[T_{n}^{(M)} X_{n}^{(M)} a(1, n|-1, \nu| p) A(n, \nu, p)\right.} \\
& \left.+T_{n}^{(N)} X_{n}^{(N)} a(1, n|-1, \nu| p, p-1) B(n, \nu, p)\right], \\
X_{\nu}^{(N)}= & -2 \pi n_{o} \sum_{n=1}^{N_{\max }} \sum_{p=|n-\nu|}^{|n+v|}(2 n+1)\left[L_{p}(k, K / b)+M_{p}(k, K / b)\right] \\
\times & {\left[T_{n}^{(M)} X_{n}^{(M)} a(1, n \mid-1, \nu / p, p-1) B(n, \nu, p)\right.} \\
& \left.+T_{n}^{(N)} X_{n}^{(N)} a(1, n|-1, \nu| p) A(n, \nu, p)\right] .
\end{aligned}
$$

The exciting field coefficients (average multipole amplitude), $X_{\nu}^{(M)}$ and $X_{\nu}^{(N)}$ are a homogeneous system of equations. The ratios of exciting field coefficients are determined by forcing the determinant to zero, and the propagation constant $K$ is solved numerically by searching for the roots of the determinant by using Muller's method. Details can be found in [20].

The effective propagation constant and the ratios of exciting field coefficients 
are substituted into Ewald-Oseen theorem [20],

$$
K-k=-\frac{\pi i n_{o}}{k^{2}} \sum_{n=1}^{N_{\max }}\left(T_{n}^{(M)} X_{n}^{(M)}+T_{n}^{(N)} X_{n}^{(N)}\right)(2 n+1),
$$

to obtain a single set of exciting field coefficients.

The absorption coefficient is calculated by using the coherent exciting field and is given as $[29]$

$$
\begin{aligned}
\kappa_{a} & =\frac{2 \pi}{k K_{r}|1-R|^{2}} n_{o} \sum_{n=1}^{N_{\max }}(2 n+1) \\
& \times\left[\left|X_{n}^{(M)}\right|^{2}\left(-\operatorname{Re} T_{n}^{(M)}-\left|T_{n}^{(M)}\right|^{2}\right)+\left|X_{n}^{(N)}\right|^{2}\left(-\operatorname{Re} T_{n}^{(N)}-\left|T_{n}^{(N)}\right|^{2}\right)\right]
\end{aligned}
$$

where

$$
\begin{aligned}
R & =\frac{\pi i n_{o}}{k^{2}\left(k+K_{r}\right)} \sum_{n=1}^{N_{\max }}(-1)^{n}(2 n+1) \\
& \times\left[-T_{n}^{(M)} X_{n}^{(M)}+T_{n}^{(N)} X_{n}^{(N)}\right] .
\end{aligned}
$$

The phase matrix for dense media radiative transfer theory is in Appendix A. The scattering coefficient is computed by using [29],

$$
\kappa_{s}=\pi \int_{0}^{\pi} d \Theta\left(P_{11}(\Theta)+P_{22}(\Theta)\right) \sin \Theta
$$

where $P_{11}(\Theta)$ and $P_{22}(\Theta)$ are the first two diagonal components of the phase 
matrix. The extinction coefficient due to the particles is calculated by adding the scattering coefficient and the absorption coefficient.

\subsubsection{DMRT Equation and Its Solutions}

The extinction coefficient and phase matrix for DMRT theory were obtained in the previous section. The DMRT equation was derived from the Bethe Salpeter equation by using the correlated ladder approximation for the incoherent field as in [20]. The form of the DMRT equation is the same as the RT Equation (2.1). The effective propagation constant and effective permittivity in the second medium are used in DMRT theory instead of the propagation constant and the permittivity of the background medium. The reduced intensity and the diffuse intensity can be obtained through the same procedure as in classical RT theory. 


\subsection{Conservation of Energy}

The classical radiative transfer (RT) theory deals with the transport of energy through a medium containing randomly distributed particles. If the medium and the particles are lossless materials, the input power will be conserved and then the output power will be the same as the input. The DMRT theory based on QCA also takes into account energy conservation. The accuracy of the RT solutions and their computer program codes was verified by testing the conservation of energy as shown in Figures 2.6 and 2.7. The summation of the reflected power flux, the upward and downward reduced power flux, and the upward and downward diffuse power flux is the same as the incident power flux in both the classical RT and DMRT/QCA models as shown in Figures 2.6(a) and 2.7(a), respectively. The reduced power flux decreases with an increase in frequency and the diffuse power flux increases with frequency as shown in Figures 2.6(b) and 2.7(b). The amount of decrease in the reduced power flux is the same as the amount of increase in the diffuse power flux since the reflected power flux does not change with frequency. 
The power flux from the incident intensity, reflected intensity, reduced intensity, and diffuse intensity are as follows.

$$
\begin{gathered}
F_{i}=\int_{0}^{2 \pi} \int_{0}^{1} \sum_{\alpha} I_{i}^{\alpha} \mu_{0} d \mu_{0} d \phi_{0}, F_{r}=\int_{0}^{2 \pi} \int_{0}^{1} \sum_{\alpha} I_{r}^{\alpha} \mu_{0} d \mu_{0} d \phi_{0}, \\
F_{r i}=\int_{0}^{2 \pi} \int_{0}^{1} \sum_{\alpha} I_{r i, 0}^{+, \alpha} \mu_{0} d \mu_{0} d \phi_{0}+\int_{0}^{2 \pi} \int_{0}^{1} \sum_{\alpha} I_{r i, 2}^{-, \alpha} \mu_{2} d \mu_{2} d \phi_{2}, \\
F_{d}=\int_{0}^{2 \pi} \int_{0}^{1} \sum_{\alpha} I_{d, 0}^{+, \alpha} \mu_{0} d \mu_{0} d \phi_{0}+\int_{0}^{2 \pi} \int_{0}^{1} \sum_{\alpha} I_{d, 2}^{-, \alpha} \mu_{2} d \mu_{2} d \phi_{2},
\end{gathered}
$$

where $\alpha$ is a superscript denoting one of the four parameters of a Stokes vector, $v, h, U$, and $V$; and $\mu=\cos \theta$. The subscripts 0 and 2 denote region 0 and region 2 , respectively. 


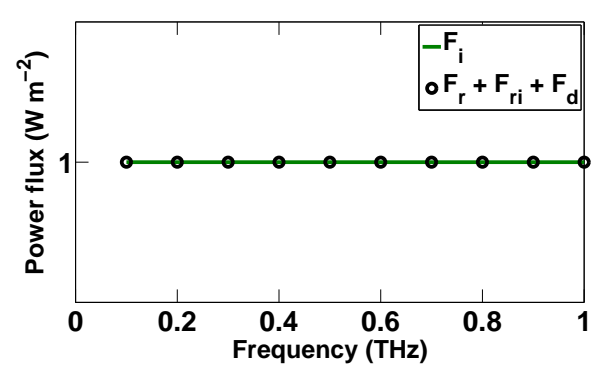

(a) $F_{i}$

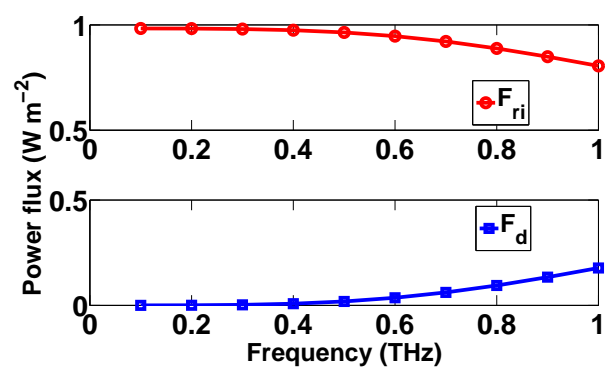

(b) $F_{r i}$ and $F_{d}$

Figure 2.6: Conservation of power in classical RT model: (a) incident power flux $\left(F_{i}\right)$ and the summation of reflected power flux $\left(F_{r}\right)$, reduced power flux $\left(F_{r i}\right)$, and diffuse power flux $\left(F_{d}\right)$, and (b) (upper) reduced power flux and (lower) diffuse power flux for $f_{v}=5 \%, a=50 \mu \mathrm{m}, d=5 \mathrm{~mm}, n_{0}=1, n_{1}=1.3, n_{2}=1$, $n_{p}=1.5$, and normal incidence $\left(\theta_{i}=0^{\circ}\right)$.

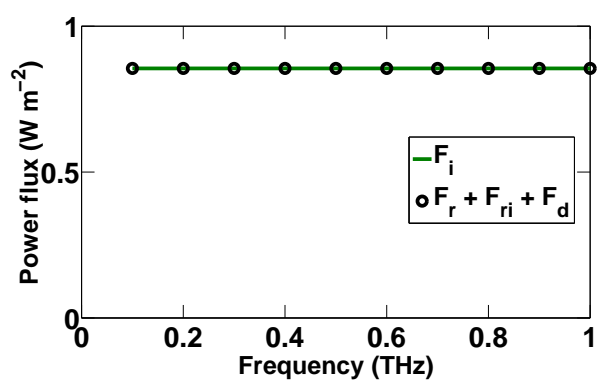

(a) $F_{i}$

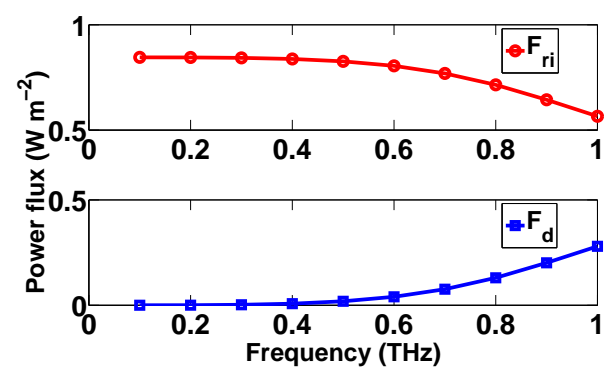

(b) $F_{r i}$ and $F_{d}$

Figure 2.7: Conservation of power in DMRT based on QCA model: (a) incident power flux $\left(F_{i}\right)$ and the summation of reflected power flux $\left(F_{r}\right)$, reduced power flux $\left(F_{r i}\right)$, and diffuse power flux $\left(F_{d}\right)$, and (b) (upper) reduced power flux and (lower) diffuse power flux for $f_{v}=20 \%, a=50 \mu \mathrm{m}, d=5 \mathrm{~mm}, n_{0}=1$, $n_{1}=1.3, n_{2}=1, n_{p}=1.5$, and oblique incidence $\left(\theta_{i}=31.21^{\circ}\right)$. 


\section{Chapter 3}

\section{Terahertz Measurement and Results}

In this chapter, experimental data will be compared with simulation results to validate the RT models. The diffuse scattered field in both transmission and reflection modes were measured using a THz-TDS system. The classical RT model for plane-parallel medium was used to simulate the diffuse specific intensity.

\subsection{Sample Preparation}

Two samples were prepared for diffuse measurements as shown in Table 3.1. The material for the background medium was Polyethylene (PE) powder whose size is less than $10 \mu \mathrm{m}$ in diameter. Steel spheres were used as scatterers and their size is $1 \mathrm{~mm}$ in diameter. The PE powder containing steel spheres was pressed with a Specac hydraulic press at 1 ton for 1 minute, resulting in pellet samples with a diameter of $4 \mathrm{~cm}$ as shown in Figure 3.1.

\begin{tabular}{|c||c|c|}
\hline & $\begin{array}{c}\text { Fractional } \\
\text { volume }(\%)\end{array}$ & $\begin{array}{c}\text { Thickness } \\
(\mathrm{mm})\end{array}$ \\
\hline \hline Sample 1 & 2 & 5 \\
Sample 2 & 3.2 & 9.5 \\
\hline
\end{tabular}

Table 3.1: Fractional volume of metal spheres, $f_{v}$, and the thickness, $d$, of two pellet samples 


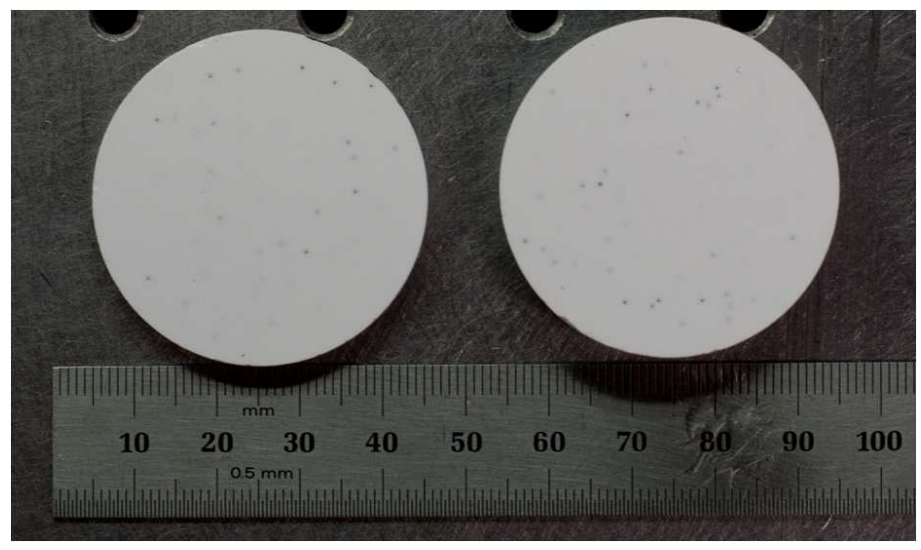

Figure 3.1: Compressed PE powder pellets containing steel spheres: sample 1 (left) and sample 2 (right).

\subsection{Measurement Process and Data Analysis}

The measurements were performed using a Picometrix T-Ray 4000 time domain spectroscopy (TDS) system. Both the transmitter and receiver used collimating lenses. The terahertz beam was vertically polarized and the receiver detected vertically polarized beams. In this experiment, 36 realizations for each sample pellet were carried out. Half of the 36 realizations were for one side of the sample and half were for the other. For the 18 realizations per side, the sample was rotated in equal angle increments through 360 degrees. Coherent averaging of multiple waveforms lowers the noise floor, increasing SNR. In these data, 10,000 time-domain waveforms were averaged for each diffuse scattering realization. 


\subsubsection{Transmission Measurements}

Figure 3.2 shows the configuration of the transmitter, the receiver, and the special sample holder in transmission mode. The sample holder was mounted to the motion stage. Transmission measurements at normal incidence were conducted at $180,160,140$, and 120 degrees from the zenith angle. A measurement without samples also was carried out at 180 degrees as a reference to normalize the data.

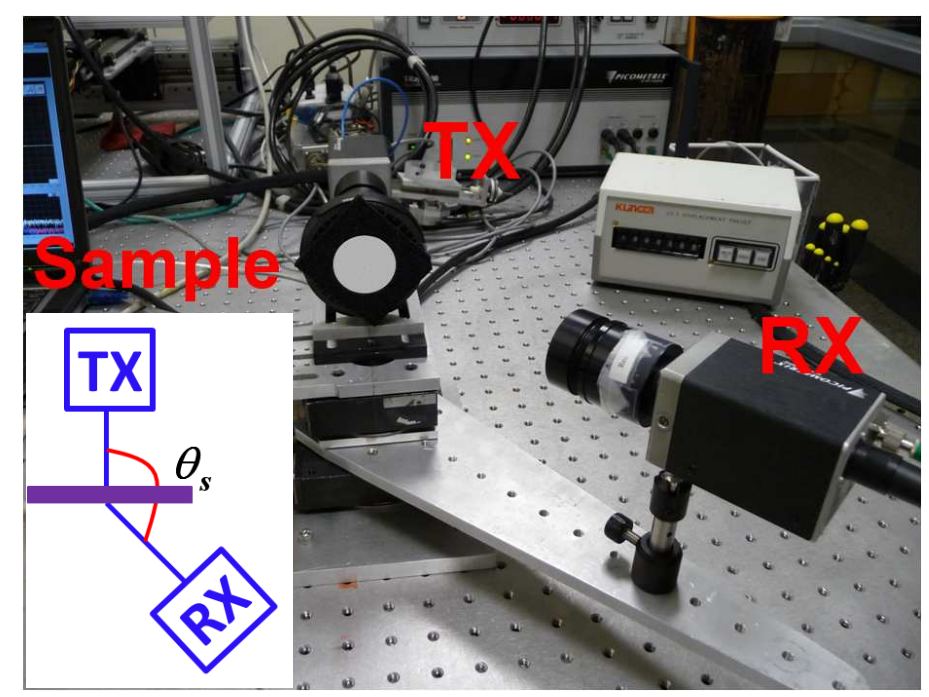

Figure 3.2: Experiment setup for transmission measurement at a normal incidence, $\theta_{i}=0^{\circ}$ and $\theta_{s}=140^{\circ}$.

Figure 3.3 shows the THz waveforms measured in transmission mode at normal incidence in the time domain. By comparing curve (a) to curve (c), we can see that the $\mathrm{THz}$ pulse was delayed and attenuated because of the sample. Since $\mathrm{PE}$ is almost transparent in the $\mathrm{THz}$ frequency range, the main cause of the attenuation is due to scattering from the steel spheres embedded in the PE 
sample. Curves (d)-(g) are 4 of the 36 diffuse scattered field realizations with each realization showing its unique waveform. The curve (b) shows the measured waveform without samples, and indicates the effect from the side lobe of $\mathrm{THz}$ beam at $\theta_{s}=160^{\circ}$.

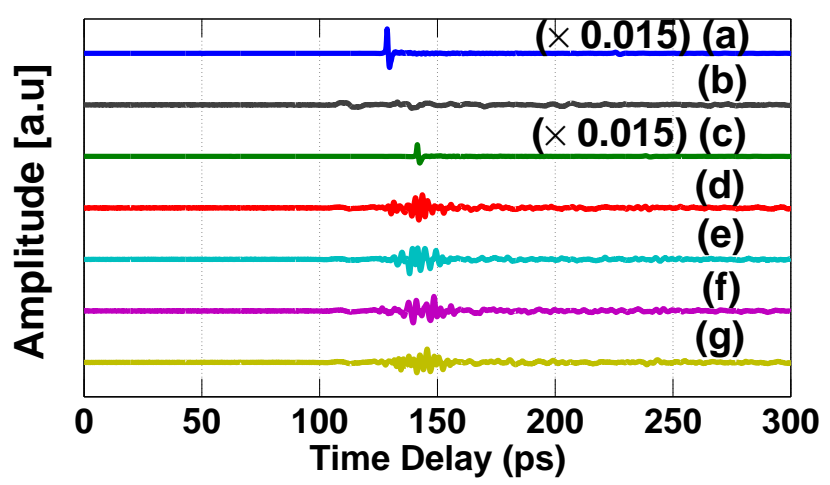

Figure 3.3: The transmission measurement at normal incidence $\left(\theta_{i}=0^{\circ}\right)$ : (a) $\mathrm{THz}$ waveforms with no sample at $\theta_{s}=180^{\circ}$ and (b) no sample at $\theta_{s}=160^{\circ}$. (c) The transmitted waves through sample 2 at $\theta_{s}=180^{\circ}$. (d) - (g) The diffuse scattered waves from sample 2 at $\theta_{s}=160^{\circ}$ (different realizations). The curves, (a) and (c) were scaled by a factor of 0.015 .

\begin{tabular}{|c||c|c|}
\hline & Sample & Scattering Angle $\left(^{\circ}\right)$ \\
\hline \hline (a) & no sample & 180 \\
(b) & no sample & 160 \\
(c) & sample 2 & 180 \\
(d)-(g) & sample 2 & 160 \\
\hline
\end{tabular}

Table 3.2: The samples used and the scattering angles observed for $\mathrm{THz}$ waveforms in Figure 3.3.

The time domain waveforms were transformed to the frequency domain by performing a fast Fourier transform (FFT). Figure 3.4 shows the THz spectra in the frequency domain that were transformed from the time domain waves from 
Figure 3.3. The diffuse waveforms and their power spectral densities at different scattering angles in transmission mode have the similar trend to those in Figures 3.3 and 3.4. All the power spectral densities for diffuse scattered waves (curves (d) - (g)) show some fluctuation but they are at approximately the same level. The frequency domain spectra for the 36 realizations were averaged, but the average spectrum still showed some random fluctuation. It was not practical to measure enough realizations to completely eliminate incoherent effects and get to the mean scattered intensity. Therefore, the data was smoothed by using linear least squares and a 2nd degree polynomial model. The averaged frequency domain data was then normalized by dividing them by the reference which was measured without samples at $\theta_{s}=180^{\circ}$.

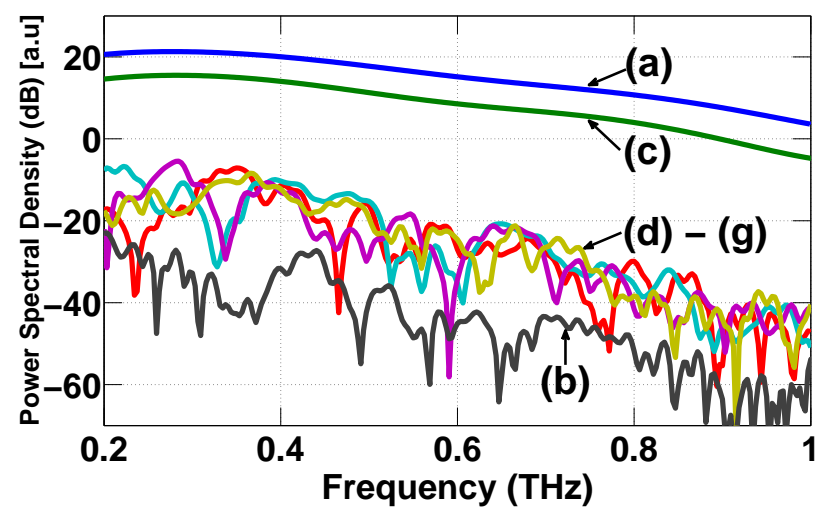

Figure 3.4: The power spectral density of the waves (a) - (g) in Figure 3.3. 


\subsubsection{Reflection Measurements}

Figure 3.5 shows the configuration of the transmitter, the receiver, and the sample holder in reflection mode. Reflection measurements with an angle of incidence of $\theta_{i}=29^{\circ}$ were taken at the specular angle $\left(\theta_{s}=29^{\circ}\right)$ and at an offspecular angle, $\theta_{s}=-6^{\circ}$. A specular reflection measurement from a smooth copper sheet was conducted $\left(\theta_{s}=29^{\circ}\right)$ and was used as the reference for the reflection measurements.

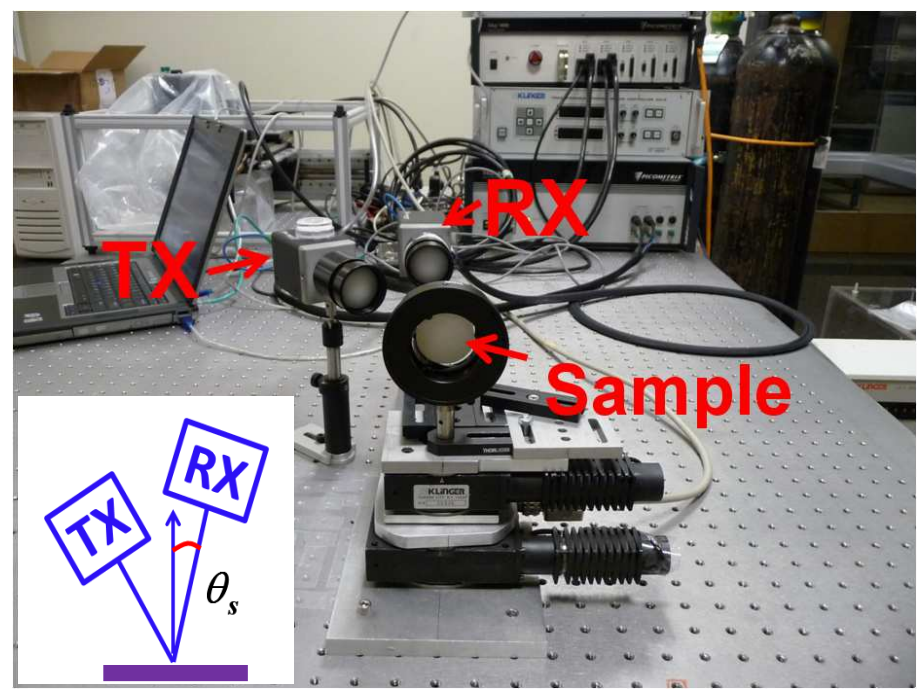

Figure 3.5: Experiment setup for reflection measurement at an oblique incidence, $\theta_{i}=29^{\circ}$ and off-specular measurement, $\theta_{s}=-6^{\circ}$.

Figure 3.6 shows the $\mathrm{THz}$ waveforms measured in reflection mode in the time domain. The curves (a) and (c) show the specular angle measurements from copper sheet and sample 1, respectively. In curve (c), the first pulse is the reflected wave which is reflected from the upper boundary surface of the sample and the 
second pulse is the reflected wave arising from the lower boundary surface. The second pulse has lower amplitude than the first pulse. Assuming there was no absorption or scattering in the background medium (PE), the attenuation is most likely because of the metal scatterers in the pathway of the coherent wave. The curves (d) - (f) are 3 of the 36 diffuse scattered field realizations, and curve (b) shows the measured waveform without samples at $\theta_{s}=-6^{\circ}$.

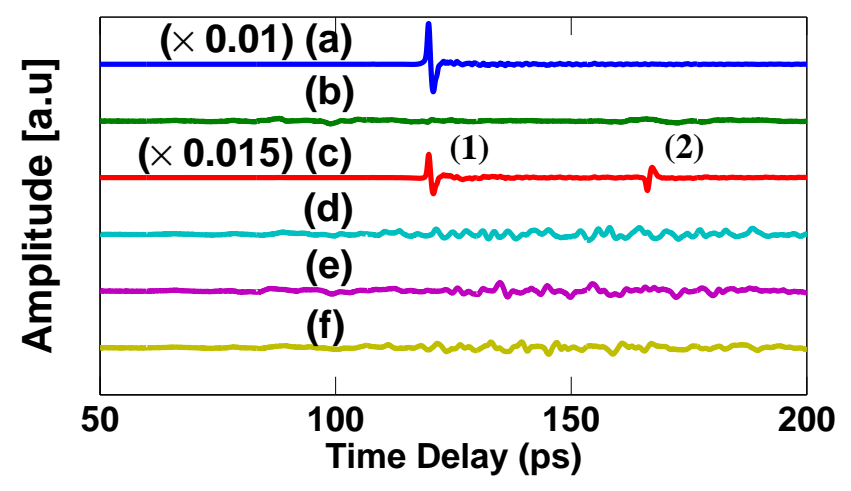

Figure 3.6: The reflection measurement at oblique incidence $\left(\theta_{i}=29^{\circ}\right)$ : (a) the specular reflection from a copper sheet $\left(\theta_{s}=29^{\circ}\right)$ and $(\mathrm{b})$ the off-specular angle $\left(\theta_{s}=-6^{\circ}\right)$ measurement from a copper sheet. (c) The reflected waves at the upper boundary (1) and at the lower boundary (2) of sample 1. Curves (d) (f) show the diffuse scattered waves for 3 of the 36 realizations from sample 1 at off-specular angle $\left(\theta_{s}=-6^{\circ}\right)$. The curve, (a) was scaled by a factor of 0.01 , and the curve, (c) was scaled by a factor of 0.015 .

\begin{tabular}{|c||c|c|}
\hline & Sample & Scattering Angle ( $)$ \\
\hline \hline (a) & copper & 29 \\
(b) & copper & -6 \\
(c) & sample 1 & 29 \\
(d)-(g) & sample 1 & -6 \\
\hline
\end{tabular}

Table 3.3: The samples used and the scattering angles observed for $\mathrm{THz}$ waveforms in Figure 3.6. 
Figure 3.7 shows the THz spectra in the frequency domain that were transformed from the time domain waves from Figure 3.6 using an FFT.

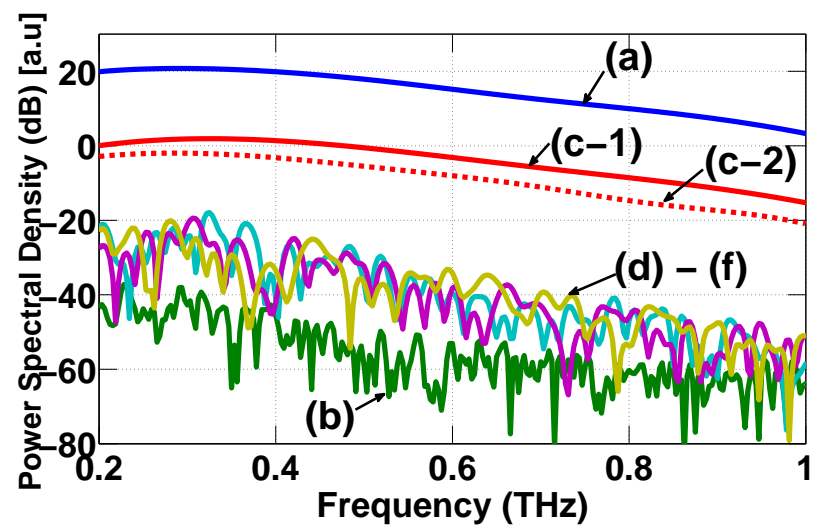

Figure 3.7: The power spectral density of the waves (a) - (f) from Figure 3.6. The curve (c-1) is the power spectral density from the first pulse and the curve (c-2) is that from the second pulse in time domain waveforms for sample 1.

The frequency domain spectra for the 36 realizations were averaged, and then smoothed in a manner similar to that used for the transmission measurements. The averaged frequency spectra were normalized by dividing them by the reference measurement.

\subsection{Modeled Transmission and Reflection Response}

In this section, the numerical solutions of the classical RT equation for a plane parallel slab is used to model the compressed PE pellets containing randomly distributed metal spheres. The parameters needed to calculate the specific intensity for the two samples are the sphere size with radius $a=500 \mu \mathrm{m}$, the 
refractive index of the sphere, $n_{p}$, the refractive indices of the surrounding media $n_{0}=1$ and $n_{2}=1$, and the background medium $n_{1}=1.46$. The sample thickness and fractional volume of the metal spheres are given in Table 3.1.

Because the refractive index of steel in the $\mathrm{THz}$ frequency range was not known, it was assumed to be $n_{p}=300+i 1000$ which is an approximate value for gold in $\mathrm{THz}$ frequency region from [35].

The calculated size parameter, albedo, and optical depth are in Table 3.4. The size parameter $k a$ is 1.5 to 15.3 at $0.1 \mathrm{THz}$ to $1 \mathrm{THz}$, respectively (where $k$ is the propagation constant in the background medium (PE) and $a$ is the radius of the particles). Thus, the metal spheres are in the Mie regime for these experiments. The albedo, which is the ratio of the scattering efficiency to the extinction efficiency, is approximately 0.9. The optical depth $\left(\tau=\kappa_{e} \times d\right)$ is approximately 0.33 for sample 1 , and 1 for sample 2 .

\begin{tabular}{|l||c|c|c|}
\hline & $\begin{array}{c}\text { size parameter } \\
(\mathrm{ka})\end{array}$ & $\begin{array}{c}\text { albedo } \\
\left(Q_{\text {sca }} / Q_{\text {ext }}\right)\end{array}$ & $\begin{array}{c}\text { optical depth } \\
\tau=\kappa_{e} \times d\end{array}$ \\
\hline \hline sample 1 & $3.1-15.3$ & & 0.33 \\
sample 2 & $($ at $0.2-1 \mathrm{THz})$ & 0.9 & 1 \\
\hline
\end{tabular}

Table 3.4: Size parameter, albedo, and optical depth for samples

Diffuse scattering from air voids in the PE powder must also be considered. Even though the PE powder is pressed and densely packed, void spaces will be created among the PE grains [10]. Assuming the air voids are spherical in shape, 
the pellet sample will consist of $20 \%$ of air voids and $80 \%$ of Teflon (See details in [10]). The air void radius $r_{v}$ is calculated using [10]

$$
r_{v}=\left[\frac{1}{\sqrt{2} \pi}-\frac{1}{6}\right]^{1 / 3} r
$$

where $r$ is the radius of a grain. The PE grains with a radius of $5 \mu \mathrm{m}$ gives corresponding air void radius of $1.96 \mu \mathrm{m}$. Air voids are further assumed to be scatterers embedded in a homogeneous PE background medium. As the PE grains are assumed to be packed tightly, the air voids between them are assumed to be small $1.96 \mu \mathrm{m}$ in radius. Figure 3.8 shows the diffuse specific intensity from metal spheres in sample 1 and from air voids. The minimum contrast is approximately $30 \mathrm{~dB}$ which is much higher than SNR in the THz TDS measurement system. Therefore, the scattering effect from air voids between the PE grains can be ignored. This is also the case for sample 2 because it has a higher fractional volume of metal spheres than sample 1.

The scattering angles chosen from the RT calculations were $\theta_{s}=159.9^{\circ}$, $\theta_{s}=139.7^{\circ}$, and $\theta_{s}=120.2^{\circ}$ in transmission measurements and $\theta_{s}=-6.2^{\circ}$ in reflection measurement which are closest to the scattering angles chosen for the measurements.

The specific intensity calculated from the numerical simulation was converted into intensity in order to compare theoretical results to measured results by using 


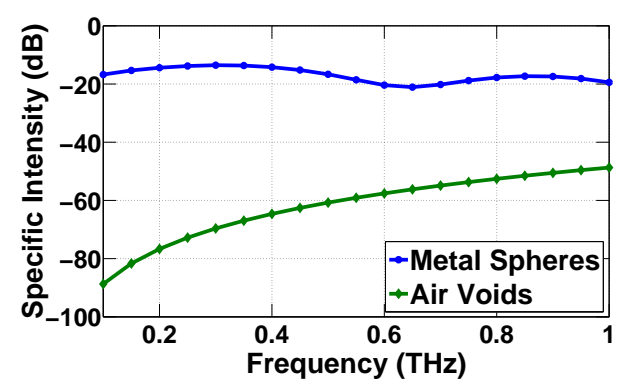

(a) Transmission Mode

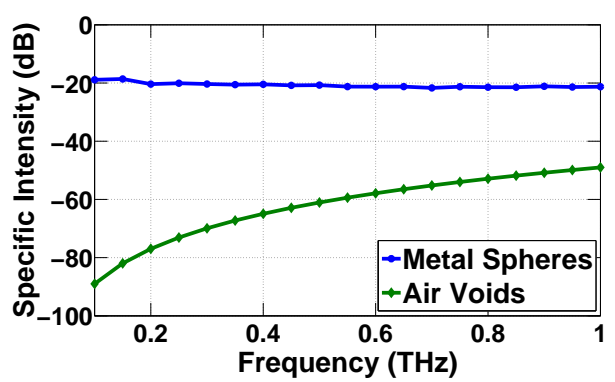

(b) Reflection Mode

Figure 3.8: Results calculated from the RT model: (a) contrast between the diffuse specific intensity from metal spheres in sample 1 and from air voids $\left(f_{v}=20 \%, a=2 \mu \mathrm{m}\right)$ in transmission mode $\left(\theta_{i}=0^{\circ}\right.$ and $\left.\theta_{s}=139.7^{\circ}\right)$, and (b) contrast between the diffuse specific intensity from the metal spheres in sample 1 and that from the air voids in reflection mode $\left(\theta_{i}=29^{\circ}\right.$ and $\left.\theta_{s}=-6.2^{\circ}\right)$.

the following formula [36]:

$$
I=\Delta \Omega I_{d} \approx \pi \theta_{d}^{2} I_{d}
$$

where $\Delta \Omega$ is the detector's field of view (FOV), and the detector's half angle beam width, $\theta_{d}$, is assumed to be small. The detector's half angle beam width is on the order of

$$
\theta_{d} \propto \frac{\lambda}{2 D}
$$

where $D$ is a linear dimension of a receiver and $\lambda$ is a wavelength. Equation (3.3) comes from the far-field beam width (full-width at half-maximum, FWHM) for a radiator of linear dimension $D$ using reciprocity theorem. The detector's field 
of view can be calculated using Equations (3.2) and (3.3)

$$
\Delta \Omega \approx \pi \theta_{d}^{2} \approx \pi\left(\frac{\lambda}{2 D}\right)^{2} \approx \pi\left(\frac{c}{2 D f}\right)^{2}
$$

where $c$ is the speed of light and $f$ is a frequency. The detector's half angle beam width was approximated to be $4^{\circ}$ at $0.1 \mathrm{THz}$ and the $\mathrm{FOV}$ is scaled to $1 / f^{2}$ as frequency increases.

\subsection{Results and Discussion}

Figure 3.9(a) shows the comparison between the normalized intensity for the measured data and that for the simulation results in transmission mode. For sample 1 , the measured intensity is approximately $1 \mathrm{~dB}$ lower than the simulated intensity, and for sample 2, the measurement is approximately $2 \mathrm{~dB}$ lower than the simulation. These differences are relatively small relative to comparisons for RT theory in the literature [36].

Sample 2 has stronger attenuation than sample 1, which results in stronger diffuse intensities in sample 2 than the diffuse intensities in sample 1 at $\theta_{s}=160^{\circ}$, $\theta_{s}=140^{\circ}$, and $\theta_{s}=120^{\circ}$ as shown in Figures 3.9(b), 3.9(c), and 3.9(d), respectively. Both measured data and simulated results show these behaviors.

An interesting observation is that the diffuse intensity at $\theta_{s}=140^{\circ}$ has one distinct spectral feature as shown in Figure 3.9(c). Also, Figure 3.9(d) shows 


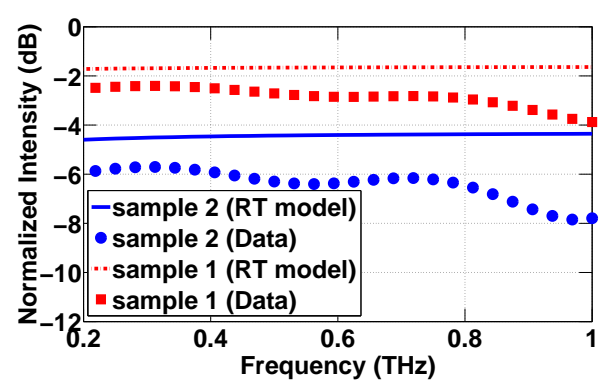

(a) At $\theta_{s}=180^{\circ}$

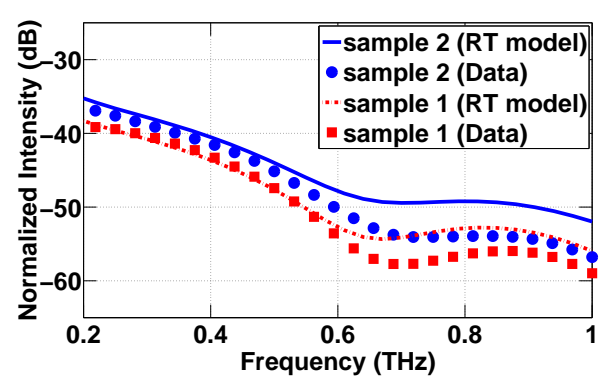

(c) At $\theta_{s}=140^{\circ}$

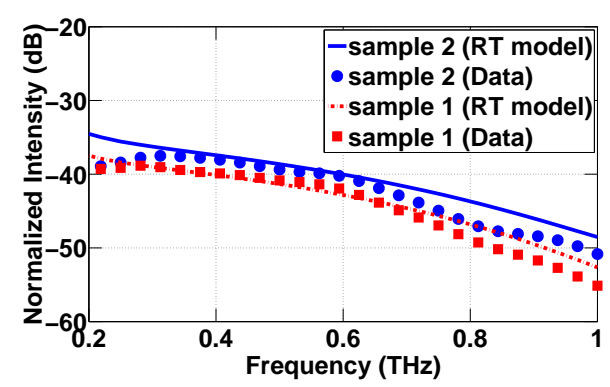

(b) At $\theta_{s}=160^{\circ}$

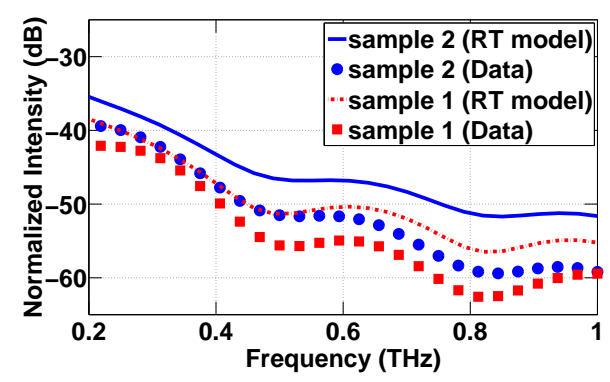

(d) At $\theta_{s}=120^{\circ}$

Figure 3.9: Comparison between the normalized intensity of measured data and simulated results in transmission mode: (a) at $\theta_{s}=180^{\circ}$, (b) at $\theta_{s}=160^{\circ}$, (c) at $\theta_{s}=140^{\circ}$, and $(\mathrm{d})$ at $\theta_{s}=120^{\circ}$.

two distinct features at $\theta_{s}=120^{\circ}$. The nulls appear in both simulations and measurements. In contrast, there are no nulls in the simulations or measurements at 180 and 160 degrees. The nulls might be due to Mie scattering of the steel spheres.

Figures 3.9(c) and 3.9(d) show that the contrast between the measured data and simulation results is sensitive to scattering angle. As the scattering angle decreases from $\theta_{s}=140^{\circ}$ to $\theta_{s}=120^{\circ}$, the contrast increases. Also the contrast is stronger at high frequencies. Possible causes for these differences will be considered at the end of this section. 
Figure 3.10(a) illustrates the specular intensity for sample 1 in reflection mode. The reflected intensity is the power spectral density of the first pulse in the time domain in Figure $3.6(\mathrm{C}-1)$, which is the bounced wave at the upper boundary surface. The reduced intensity is the power spectral density of the second pulse in the time domain in Figure 3.6 (C-2), which is the bounced wave at the lower boundary surface once but not multiple times. The measured reflected intensity is approximately $3 \mathrm{~dB}$ lower than the simulated intensity, and the measured reduced intensity is approximately $4 \mathrm{~dB}$ lower than the simulation, which are relatively small.

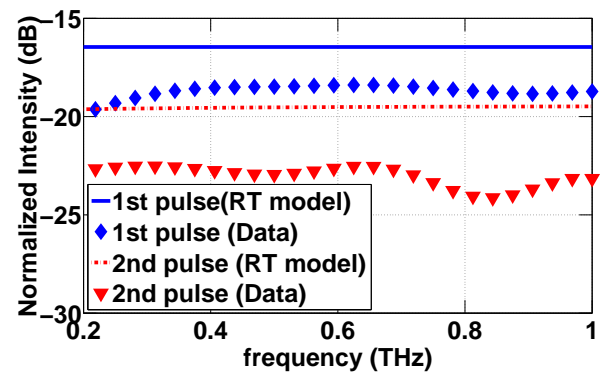

(a) At specular angle

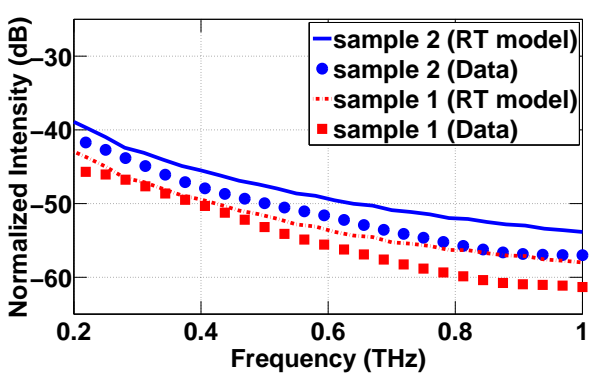

(b) At $\theta_{s}=-6^{\circ}$

Figure 3.10: Comparison between the normalized intensity of measured data and simulated results in reflection mode: (a) (1st pulse) the reflected intensity bounced at the upper boundary of sample 1 and (2nd pulse) the summation of the reduced intensity and diffuse intensity at specular angle. The reduced intensity was bounced at the lower boundary once, but multiple times. The diffuse intensity is negligible. (b) The diffuse intensity at off-specular angle $\theta_{s}=-6^{\circ}$.

Figure 3.10(b) shows the comparison between the measurement and simulation at off-specular angle in reflection mode. The experimental data is a few dB 
lower than the simulation results. The diffuse intensity for sample 2 is stronger than sample 1 in both the experimental and simulated results. This is most likely caused by the higher fractional volume of metal spheres in sample 2 .

In conclusion, the RT model predicted that the reduced intensity for sample 2 (higher fractional volume of scatterers and thicker layer) attenuates more strongly than that for sample 1 (lower fractional volume and thinner layer) and the measured data showed the same results. Also, the RT model predicted the diffuse intensities for sample 2 are stronger than those for sample 1 and the measurements had the same results. Moreover, in both measurements and simulations, one null at $\theta_{s}=140^{\circ}$ and two nulls at $\theta_{s}=120^{\circ}$ were shown in transmission mode.

In general the RT simulations for diffuse intensity showed relatively good agreement with the levels and trends observed in the measurements. However, there was some small disagreement between the measurements and simulations which increased with scattering angle and frequency. The exact cause for these behaviors is not known at this time, but may be due to the way the experiment was modeled. The calculations in Section 3.3 assumed that the PE grains were packed as tightly as possible. Experimentally, this was accomplished by pressing the PE grains together with a hydraulic press. However, the amount of pressure and time needed to tightly compress the PE grains is unknown. Perhaps the size 
and/or fractional volume of the air voids in the samples was sufficient to cause significant scattering at higher frequencies. The techniques necessary to tightly pack powder grains was not the primary topic of this research and remains an open topic for future work.

In addition, in the model, the incident beam is an infinite plane wave, but in the measurement, the incident beam is a finite quasi-Gaussian wave and the beam size and distribution are frequency-dependent. The height and width of main lobe and side lobes are also frequency-dependent. Also, in the model, an infinite size of a slab of a homogeneous medium containing scatterers is assumed, but in the measurement, the samples were a finite size with a diameter of $4 \mathrm{~cm}$. Finally, in the model, the detector is supposed to be a point detector which detects a diffuse specific intensity in the observed direction, but in the measurement, the size of the receiver was bigger than a point detector. It should be noted that these differences are not related to the RT model itself, but to assumptions about unknown parameters in the experimental setup.

Even though there were some small differences with experimental results, the RT model is validated with the qualitative experimental results. Future work could improve the experimental conditions to much closer to the ideal RT model to compare the simulation and measurement more accurately. 


\section{Chapter 4}

\section{Simulation Results}

This chapter presents simulation results for diffuse scattering from granular media using the RT theories presented in Chapter 2. The diffuse scattering is governed by various factors such as the material properties of the particles, their sizes, and concentration (number of particles per unit volume) in the medium, the material properties of the background media, the thickness of the medium, the surrounding media, and the incident wavelength. The diffuse intensity from volume scattering in granular media is investigated through simulations by varying these parameters supplied to the RT model.

\subsection{Simulations of Diffuse Specific Intensity}

The parameters of three materials, Teflon (polytetrafluroethylene: PTFE), lactose (milk sugar), and composition-4 (C4) explosive grains are used for the discrete random media in these RT simulations. Teflon is nearly transparent in the $\mathrm{THz}$ band, i.e. there is no absorption of electromagnetic radiation, while lactose and $\mathrm{C} 4$ have spectral absorption signatures in the $\mathrm{THz}$ frequency range. The parameters of the three granular media used to simulate the diffuse specific intensity from volume scattering are shown in Table 4.1. 
In these simulations, an important condition is that the parameter size, $k a$, is too low to exhibit Mie resonance effects in the frequency spectra.

In the simulations for medium 1, the relationship between diffuse intensity and factors such as grain size, concentration, and layer thickness is investigated. Also, the angle dependence of the diffuse intensity is investigated. In the simulations corresponding to media 2 and 3 , the relationship between the material properties of the grains and the diffuse intensity is investigated by varying the grain size, concentration, and layer thickness.

The diffuse specific intensity for medium 1 is calculated using the DMRT model based on QCA. For media 2 and 3, the diffuse intensity is calculated using the classical RT model for low fractional volume of scatterers (5\%) and the DMRT model based on QCA for high fractional volume (20\%). The numerical calculation is performed at 32 angular positions in the range $0^{\circ} \leq \theta \leq 180^{\circ}$. Both incident and scattered waves are assumed to have vertical polarization. The incident angles are $\theta_{0 i}=31.21^{\circ}$ and $\phi_{0 i}=0^{\circ}$, and the scattering angles are $\theta_{0 s}=31.21^{\circ}$ and $\phi_{0 s}=180^{\circ}$ in reflection mode, which is backscattering, and $\theta_{2 s}=148.79^{\circ}$ and $\phi_{2 s}=180^{\circ}$ in transmission mode, respectively, as shown in Figure 4.1. 


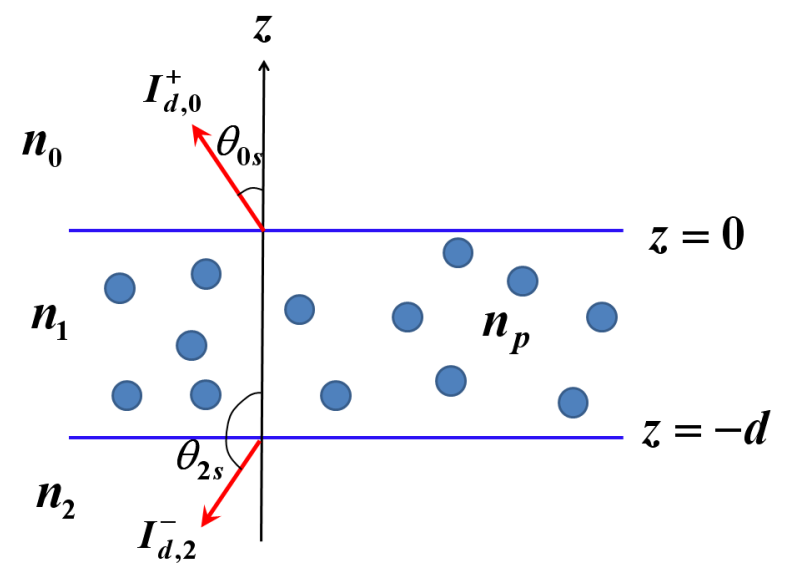

Figure 4.1: Geometrical configuration assumed in RT simulation.

\begin{tabular}{|c||c|c|c|c|c|c|}
\hline & $n_{0}, n_{2}$ & $n_{1}$ & $n_{p}$ & $a(\mu \mathrm{m})$ & $f_{v}(\%)$ & $d(\mathrm{~mm})$ \\
\hline \hline Medium 1 & 1 & Teflon & 1 & $2,38.8,100,200$ & $1,2,4,8,16,20$ & $5,20,100$ \\
Medium 2 & 1 & 1.30 & lactose & $25,50,100$ & 5,20 & 5,10 \\
Medium 3 & 1 & 1.46 & $\mathrm{C} 4$ & $25,50,100$ & 5,20 & 5,10 \\
\hline
\end{tabular}

Table 4.1: Parameters for granular media used in simulations.

\subsubsection{Simulation Results for Medium 1}

Medium 1 consists of air voids in Teflon background. Teflon has low absorption in $\mathrm{THz}$ frequency range and its refractive index, $n_{p}=1.433$, is nearly constant across frequency [18]. A slab of pressed Teflon grains with plane-parallel top and bottom surfaces will contain air voids due to the granular structure of the Teflon [10]. These air voids are assumed to be spherical scatterers embedded in a homogeneous Teflon background medium.

The diffuse specific intensities from the Teflon slabs were calculated by varying the size of air voids and the thickness of the slabs. First, the diffuse specific 
intensities from the Teflon slabs for varying air void sizes were obtained and shown in Figures 4.2(a) and 4.2(b) for reflection and transmission mode, respectively.

The diffuse specific intensity for the smallest air voids $(2 \mu \mathrm{m})$ increases with frequency in both reflection and transmission mode. Since the fractional volume of air voids in this medium is $1 \%$, the dense media RT formulation will converge to the classical RT formulation. In addition, the smallest air void is in the Rayleigh regime because the size parameter $k a=0.06$ at $1 \mathrm{THz}$. Thus, its scattering coefficient will be proportional to $k^{4} a^{6}$. The figures show that the diffuse intensity also has this dependency: the rate of increase is proportional to the fourth power of the frequency.

In the diffuse specific intensity for $100 \mu \mathrm{m}$ air voids, the rate of increase as a function of frequency is lower than for Rayleigh scattering at high frequencies. For the $200 \mu \mathrm{m}$ air voids, the diffuse intensity increases a little at low frequencies and decreases at high frequencies. These effects are due to the dependence of scattering on the size parameter.

Next, the relationship between the thickness of the scattering layer and the diffuse intensity is shown in Figures 4.3(a) and 4.3(b) for reflection and transmission mode, respectively. Figure 4.3(a) illustrates that a thicker layer increases 


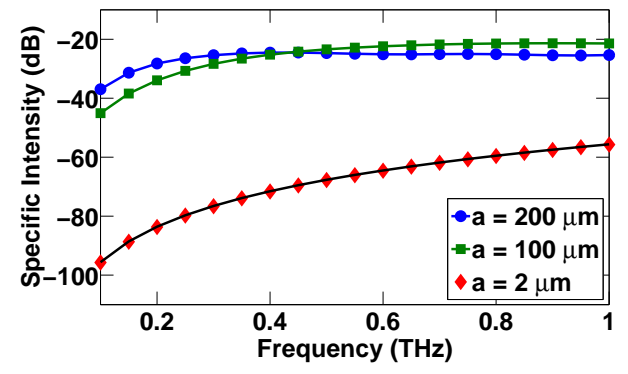

(a) Reflection Mode

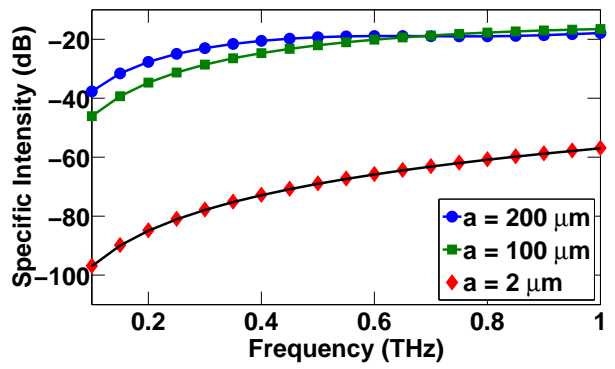

(b) Transmission Mode

Figure 4.2: Diffuse specific intensity from medium 1 obtained by varying the size of air voids: $d=5 \mathrm{~mm}$ and $f_{v}=1 \%$. (black curve) The curve is proportional to $f^{4}$.

diffuse scattered intensity in reflection, but at high frequencies the diffuse intensity increases more gradually with frequency. Figure 4.3(b) shows that the diffuse intensity increases with slab thickness at low frequencies but it decreases with the slab thickness at high frequencies in transmission mode. This indicates that less energy is able to go all the way through the thick slab. Since the extinction coefficients $\left(\kappa_{e}\right)$ for three cases are the same, the attenuation of the reduced intensity depends solely on the slab thickness. As a result, the reduced intensity for $d=10 \mathrm{~cm}$ is lower than that for $d=2 \mathrm{~cm}$. According to conservation of energy, the diffuse intensity for $d=10 \mathrm{~cm}$ must be higher than that for $d=2 \mathrm{~cm}$ because the media are lossless.

Figure 4.4 illustrates that the diffuse intensity for thickness of $d=10 \mathrm{~cm}$ increases in all directions in reflection mode, and decreases in transmission mode. However, the added diffuse intensity from the thicker layer in all directions is 
higher than that from a thinner layer. The figure also shows diffuse specific intensity depends on scattering angle.

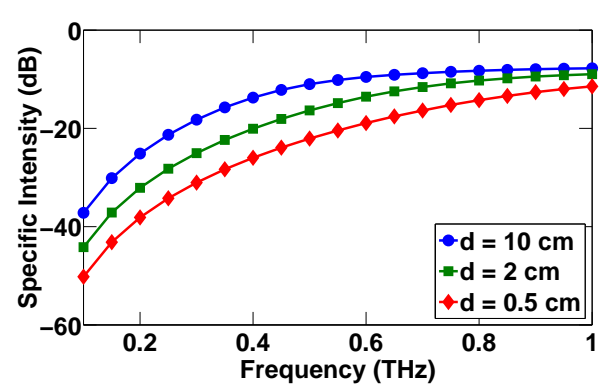

(a) Reflection Mode

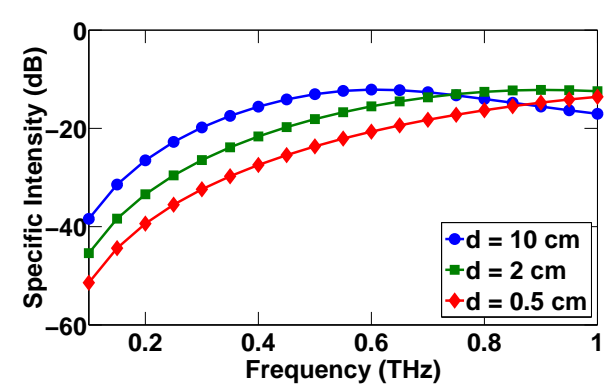

(b) Transmission Mode

Figure 4.3: Diffuse specific intensity from medium 1 obtained by varying the thickness $d$ of the medium: $a=38.8 \mu \mathrm{m}$ and $f_{v}=20 \%$.

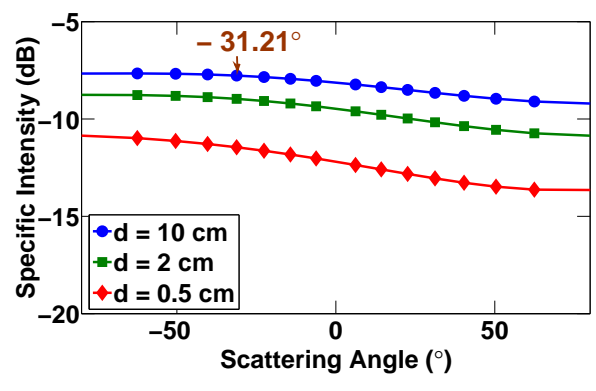

(a) Reflection Mode

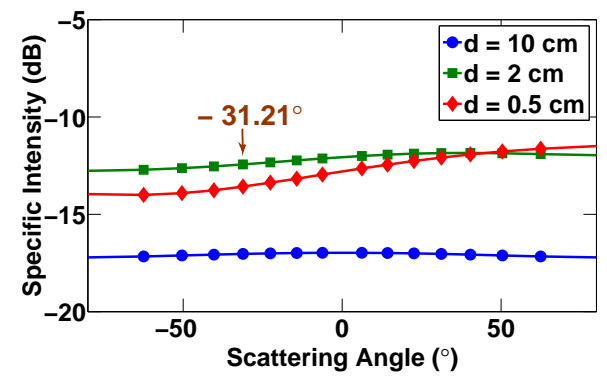

(b) Transmission Mode

Figure 4.4: Diffuse specific intensity as a function of scattering angle at $1 \mathrm{THz}$. As in Figure 4.3, $a=38.8 \mu \mathrm{m}$ and $f_{v}=20 \%$.

Figure 4.5 illustrates the dependence of diffuse intensity on the fractional volume of air voids. As shown in the figure, the diffuse specific intensity increases linearly as a function of the fractional volume at low fractional volume but it does not at high fractional volume. One reason is that the extinction rate is not proportional to the fractional volume, as shown in Figure 2.4 in Chapter 2. 
Another explanation comes from the scattering angle dependence of the diffuse intensity.

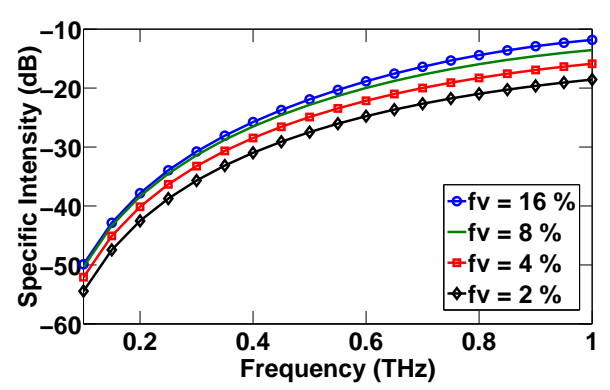

(a) Reflection Mode

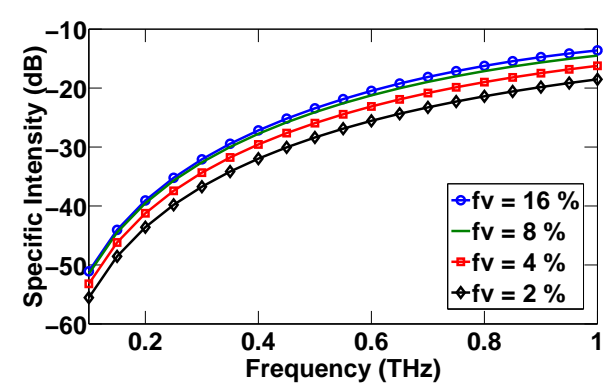

(b) Transmission Mode

Figure 4.5: Diffuse specific intensity from medium 1 obtained by varying the fractional volume of the air voids: $a=38.8 \mu \mathrm{m}$ and $d=5 \mathrm{~mm}$.

In conclusion, diffuse intensity changes as the particle size, fractional volume, or thickness of scattering layer changes, but their relationship is not linear. In addition, diffuse intensity depends on observation angle.

\subsubsection{Simulation Results for Medium 2}

Medium 2 consists of lactose grains in the background medium with refractive index of 1.30. Unlike Teflon, lactose has sharp spectral signatures in the THz frequency range. The real part of its permittivity is also much greater than its imaginary part $\left(\epsilon^{\prime}>>\epsilon^{\prime \prime}\right)$ as shown in Figure $4.6[37,38]$. Two minima can be observed in the real permittivity of lactose at 0.56 and $1.39 \mathrm{THz}$, while two maxima can be observed in the imaginary permittivity of lactose at 0.53 and $1.36 \mathrm{THz}$. 

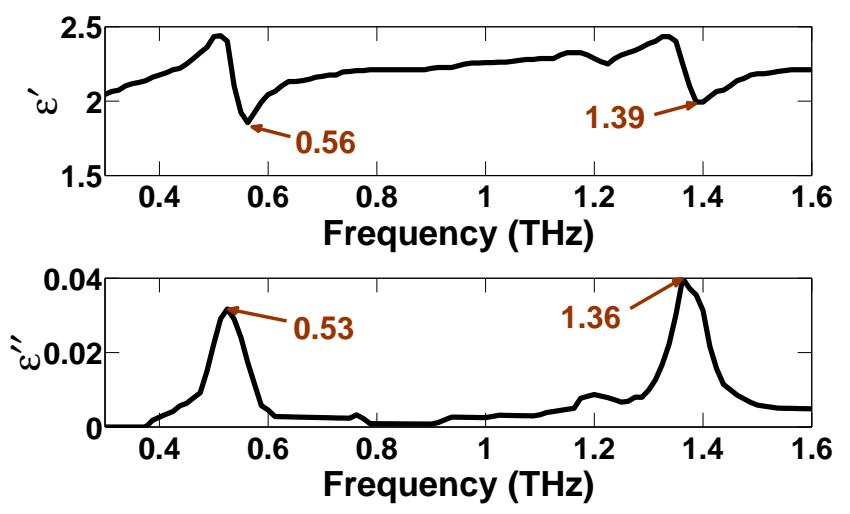

Figure 4.6: Lactose permittivity: real part $\epsilon^{\prime}$ (upper curve) and imaginary part $\epsilon^{\prime \prime}$ (lower curve) [37, 38].

Figure 4.7 shows the diffuse specific intensities obtained in reflection mode with the parameters for medium 2 in Table 4.1. As the size of lactose grains increases, the diffuse specific intensity increases for low frequencies. In contrast, particle size has a decreasing effect on diffuse specific intensity at high frequencies. This trend is similar to that of medium 1 whose increasing rate of specific intensity as a function of frequency is low at high frequencies, however, the two narrow spectral features of lactose are shown at $0.56 \mathrm{THz}$ and $1.39 \mathrm{THz}$ unlike in the case of medium 1. These spectral features are unaffected by particle size, fractional volume, or slab thickness. In reflection mode, the spectral features of the real permittivity of lactose grains are shown in the diffuse intensity.

Figure 4.8 shows the diffuse specific intensities in transmission mode. As either the fractional volume of the grains or the thickness of the medium changes, the effect of grain size on the specific intensity changes. The two spectral features 


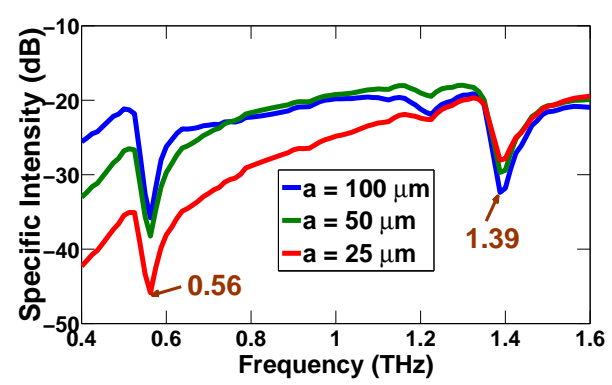

(a) $f_{v}=5 \%$ and $d=5 \mathrm{~mm}$

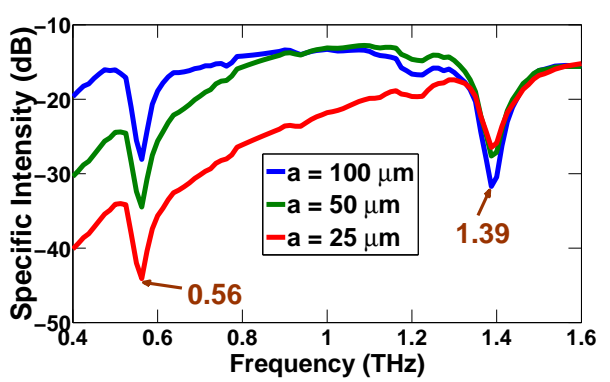

(b) $f_{v}=20 \%$ and $d=10 \mathrm{~mm}$

Figure 4.7: The diffuse specific intensities in reflection mode for medium 2 in Table 4.1.

of lactose are also shown in the transmission mode data. Interestingly, the narrow spectral feature at $1.39 \mathrm{THz}$ changes shape and is displaced slightly in frequency as the fractional volume increases for the case of $100 \mu \mathrm{m}$ lactose grains with $f_{v}=20 \%$ and $d=10 \mathrm{~mm}$ as shown in Figure $4.8(\mathrm{~b})$. The $1.39 \mathrm{THz}$ feature appears to be shifted down to $1.35 \mathrm{THz}$. This indicates that the $1.36 \mathrm{THz}$ feature in the imaginary permittivity might strongly influence the diffuse spectra and distort the $1.39 \mathrm{THz}$ feature in the real permittivity.

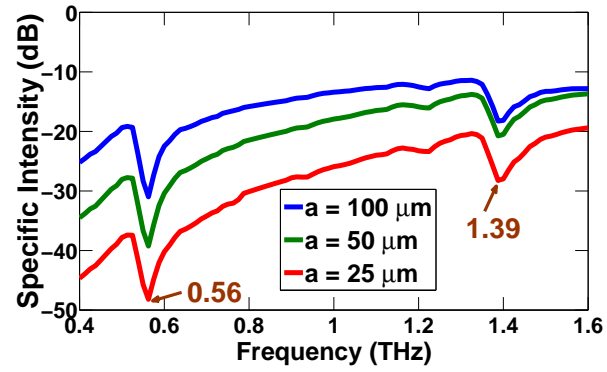

(a) $f_{v}=5 \%$ and $d=5 \mathrm{~mm}$

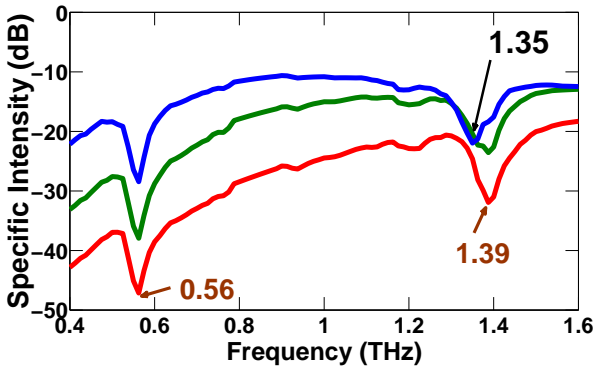

(b) $f_{v}=20 \%$ and $d=10 \mathrm{~mm}$

Figure 4.8: The diffuse specific intensities in transmission mode for medium 2 in Table 4.1.

In this medium, the spectral features of lactose are shown in the diffuse 
intensity spectra in both reflection and transmission mode, but for $100 \mu \mathrm{m}$ lactose grains with $20 \%$ of fractional volume, the high frequency spectral feature is not shown in transmission mode. The diffuse specific intensity was strongly governed by scattering effects in both reflection and transmission modes, but as the size of the lactose grains increases to $100 \mu \mathrm{m}$ and the fractional volume of grains is $20 \%$, the absorption of the large lactose grains affects the diffuse specific intensity in transmission mode, modifying the high frequency spectral feature.

\subsubsection{Simulation Results for Medium 3}

Medium 3 consists of $\mathrm{C} 4$ grains in the background medium with refractive index of 1.46. Explosive composition 4 (C4) has spectral signatures in the $\mathrm{THz}$ frequency range, but the imaginary part of its permittivity is much greater than that of lactose as shown in Figure 4.9 [39].
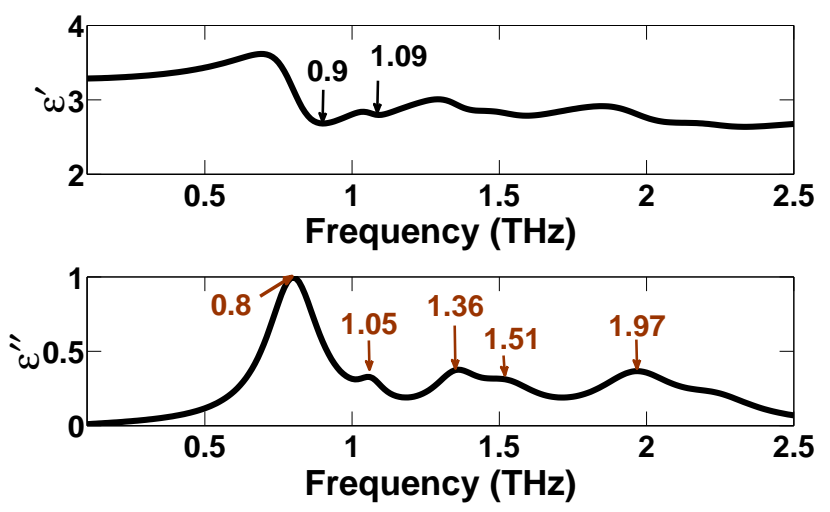

Figure 4.9: C4 permittivity: real part $\epsilon^{\prime}$ (upper curve) and imaginary part $\epsilon^{\prime \prime}$ (lower curve) [39]. 
Figure 4.10 shows the diffuse specific intensities obtained using the parameters for medium 3 in Table 4.1 in reflection mode. The diffuse specific intensity for each grain size has its own unique spectral shape. At high frequencies, the diffuse intensity for the smallest grain size $(25 \mu \mathrm{m})$ is strongest while at low frequencies the diffuse intensity for the largest grain $(100 \mu \mathrm{m})$ dominates. In Figure 4.10(c), the narrow features at $0.9 \mathrm{THz}$ and $1.09 \mathrm{THz}$ come from the spectral features of the real permittivity of $\mathrm{C} 4$, while the narrow features at $1.38 \mathrm{THz}$ and $1.52 \mathrm{THz}$ come from the spectral features of the imaginary permittivity. However, the $0.67 \mathrm{THz}$ feature shown in the specific intensity corresponding to the $100 \mu \mathrm{m}$ C4 grains does not correspond to a $\mathrm{C} 4$ spectral feature. These results suggest the estimation of material properties of particles in reflection mode may be complicated due to the dependence of the diffuse intensity on both scattering and absorption.

Figure 4.11 shows the diffuse specific intensities in transmission mode. In contrast to the diffuse specific intensity in reflection mode, the diffuse specific intensity for different grain sizes has similar spectra, and the overall amplitude is proportional to grain size. The degree of this grain size dependence changes with the fractional volume and the thickness of the slab. This is most clearly illustrated in the case of $f_{v}=20 \%$ and $d=10 \mathrm{~mm}$, the grain size has little effect on the diffuse intensity. The attenuation is also very high, so there are deep 


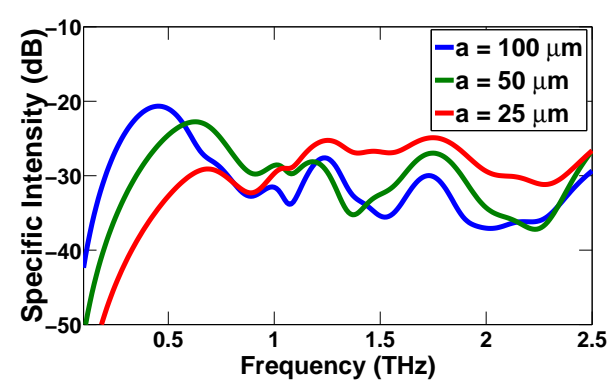

(a) $f_{v}=5 \%$ and $d=5 \mathrm{~mm}$

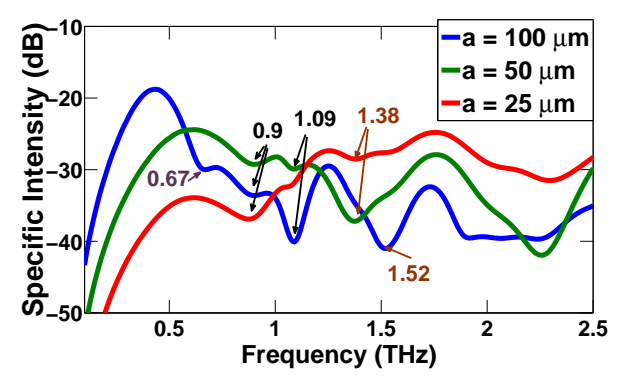

(c) $f_{v}=20 \%$ and $d=5 \mathrm{~mm}$

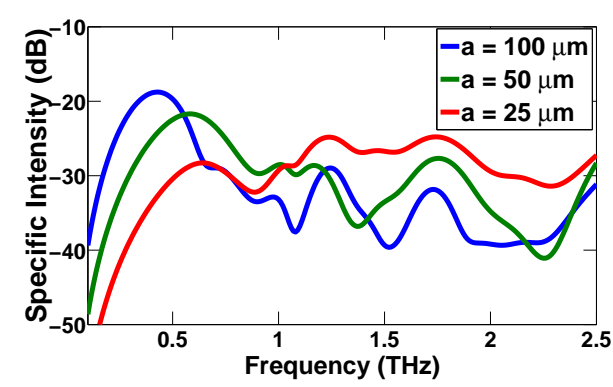

(b) $f_{v}=5 \%$ and $d=10 \mathrm{~mm}$

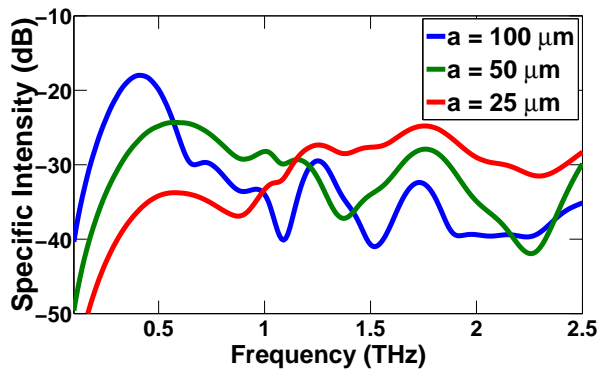

(d) $f_{v}=20 \%$ and $d=10 \mathrm{~mm}$

Figure 4.10: The diffuse specific intensities in reflection mode for medium 3 in Table 4.1.

nulls at frequencies corresponding to bands of high absorption. In particular, the sharpest null is located at $0.8 \mathrm{THz}$, corresponding to the peak of the strongest $\mathrm{C} 4$ absorption feature as shown in Figure 4.9. In transmission mode, the absorption peaks in the imaginary permittivity of $\mathrm{C} 4$ grains are shown, although some peaks are shifted by a few tens of GHz. This suggests absorption affects dominate scattering effects in this case.

In conclusion, because of strong absorption characteristics of $\mathrm{C} 4$, the diffuse spectra retains unchanged the absorption peaks of $\mathrm{C} 4$ in transmission mode. 


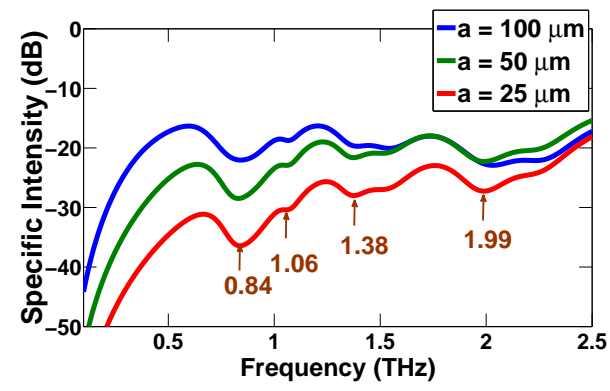

(a) $f_{v}=5 \%$ and $d=5 \mathrm{~mm}$

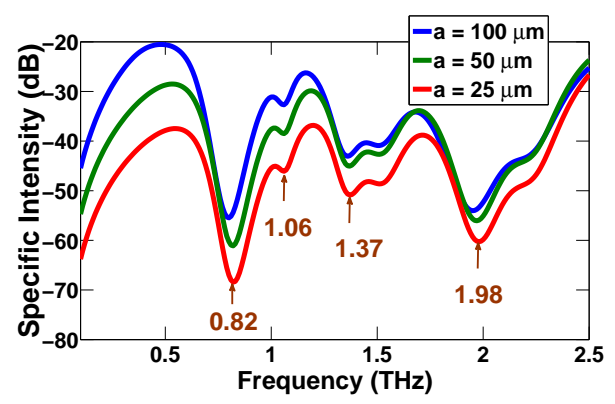

(c) $f_{v}=20 \%$ and $d=5 \mathrm{~mm}$

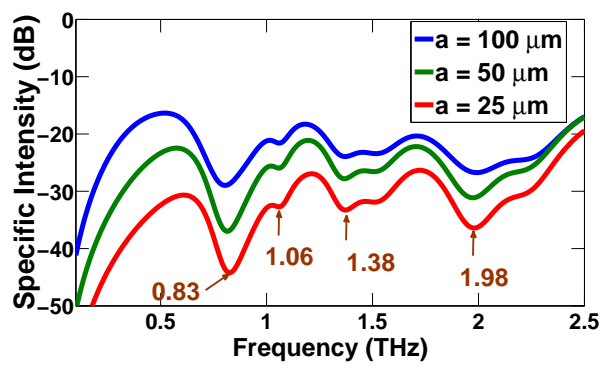

(b) $f_{v}=5 \%$ and $d=10 \mathrm{~mm}$

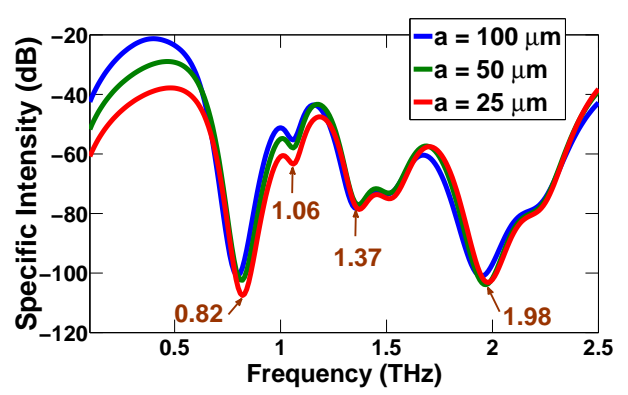

(d) $f_{v}=20 \%$ and $d=10 \mathrm{~mm}$

Figure 4.11: The diffuse specific intensities in transmission mode for medium 3 in Table 4.1.

However, the scattering characteristics in reflection mode affects the diffuse intensity and changes the spectra of the diffuse intensity. Therefore, the diffuse intensity in reflection mode for $\mathrm{C} 4$ grains shows complicated spectra due to both scattering effects and material absorption.

\subsection{Conclusion}

Table 4.2 summarizes the simulation results for the three granular media. In medium 1, there are no spectral features because the intrinsic properties of Teflon display no spectral features. In addition, the simulation results showed that the 
size of air voids, the thickness of the slab, and the fractional volume all affect the diffuse scattering. Furthermore, dependence of the diffuse intensity on scattering angle was indicated.

Medium 2 contains lactose grains which have $\mathrm{THz}$ spectral signatures, and scattering effects are much stronger than absorption in this medium. The simulation results for medium 2 show that the diffuse intensity has similar spectral features to the real permittivity of lactose due to the strong influence of scattering. However, for large particles $100 \mu \mathrm{m}$ in radius, absorption effects start to influence the transmission spectra especially at high frequencies.

Absorption is much stronger than scattering in medium 3, which contains $\mathrm{C} 4$ grains. The absorption peaks of the $\mathrm{C} 4$ grains dominate the diffuse spectra in transmission mode. However, in reflection mode, both the real permittivity and imaginary permittivity influences the diffuse spectral returns.

In conclusion, the diffuse intensity in granular media is affected by grain size and fractional volume, as well as thickness of the media and the material properties of the grains. The diffuse intensity is governed by the extinction rate (absorption + scattering) of the medium and the scattering due to the grains. The effect of absorption on transmission spectra is shown to be stronger than in reflection mode spectra. Conversely, the scattering effects in reflection mode are shown to be stronger than in transmission mode. Thus, under certain conditions 


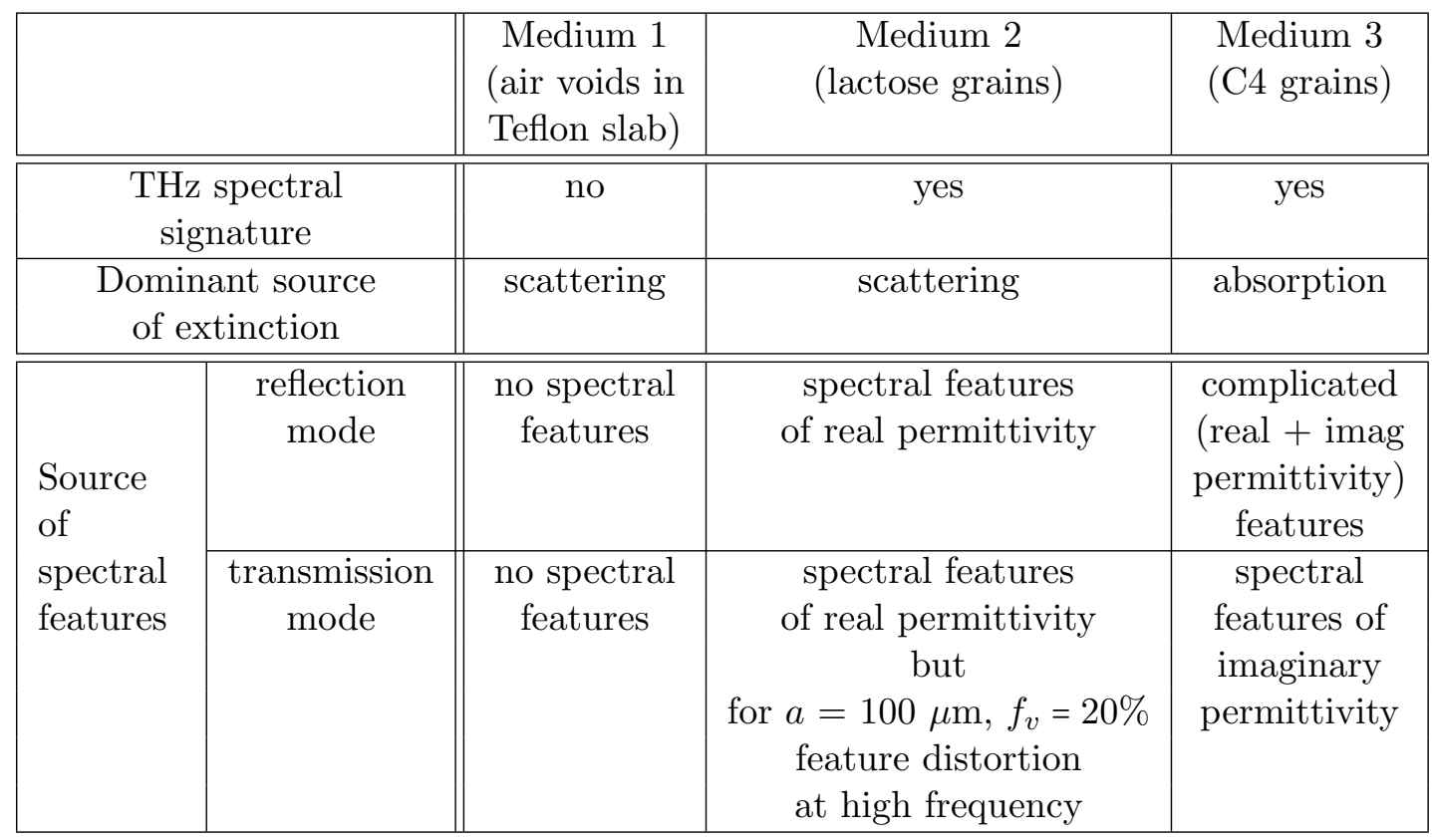

Table 4.2: Material properties of three granular media and their influence on diffuse intensity spectra

(e.g. no Mie resonances), a medium containing grains with $\mathrm{THz}$ spectral features might be able to be identified from diffuse scattering intensity. Complications may arise due to the combined influence of absorption and scattering effects.

This chapter explored the diffuse scattering from granular media using simulations based on scattering models. However, the dense media RT model software has some limits: the size parameter $k a$ is limited to approximately 2.5 and only lossless background media can be applied. Thus, an improved dense media RT model without these limitations could be used for more realistic cases such as $80 \%$ of explosive material and $20 \%$ of plastic filler and air voids in explosives. 


\section{Chapter 5}

\section{Conclusions and Future Work}

\subsection{Conclusions}

The terahertz frequency region of the electromagnetic spectrum has recently become available for scientific study and holds great promise for spectroscopic identification of materials. Earlier work studied THz wave propagation and its scattering in layered random media. Some research has also been done in the areas of rough surface scattering effects on reflection, and volume scattering effects on transmission measurements. However, until now little research has been done in the area of diffuse volume scattering. The goal of this thesis was to study $\mathrm{THz}$ diffuse scattering from layered random media. This was conducted by modeling diffuse scattering using radiative transfer (RT) theory for the first time in $\mathrm{THz}$ sensing.

Since RT theory deals with the transport of energy in random media, it is mathematically simple, and therefore practical to apply in scattering problems. Polarization and multiple scattering effects were included by using the vector radiative transfer equation and solving the equation numerically. Since RT theory (called classical RT theory) is limited to low fractional volume (less than 5\%), dense media radiative transfer (DMRT) theory based on QCA for dense media 
was applied to the case for high fractional volume. The scenarios considered in this study were limited to detection of spherical particles composed of a material of interest in a PE background, because the DMRT model assumes that lossy particles are suspended in a lossless background medium. The numerical solutions for the two RT models were implemented in MATLAB and their accuracy was validated by testing the conservation of energy.

The RT model was validated by laboratory measurements using two compressed PE pellets containing steel spheres. The measurement results showed two extreme cases of energy redistribution. For highly scattering medium, the reduced intensity is strongly attenuated. This energy is redistributed in the form of diffuse intensity, so the diffuse intensity is very strong. For weakly scattering medium, there is very little diffuse intensity and therefore the change in the reduced intensity is also small. Also, angle dependent spectral features due to Mie scattering were observed.

Diffuse volume scattering from random media containing Teflon, C4 explosive, and lactose sugar grains were investigated using RT models. The simulation results showed that the diffuse intensity was affected by the material properties of grains as well as other factors such as grain size, fractional volume of grains, thickness of scattering layer, and scattering angles. The medium containing grains with $\mathrm{THz}$ spectral features showed some spectral features in its diffuse 
intensity spectra. The absorption effect was observed in transmission mode and the scattering effect was clearly visible in reflection mode. The simulation results indicated that it is possible to identify materials from diffuse intensity spectra under certain conditions.

In this thesis, radiative transfer model was used for $\mathrm{THz}$ sensing for the first time. The numerical solutions of RT equations were implemented in MATLAB. Also, The RT model predictions were validated by measurements using compressed PE pellets containing steel spheres. Moreover, the simulation results using the RT models indicate that a medium containing grains with $\mathrm{THz}$ spectral signature might be able to be identified from diffuse intensity spectra.

\subsection{Future Work}

This thesis work was focused on only diffuse volume scattering for granular medium with planar boundaries. It could also be extended to rough surface boundaries, so the diffuse scattering from both volume scattering and surface scattering could be studied. The limitation of the DMRT model software could be obviated by extending the validation from the parameter size $(k a \approx 2.5)$ to high parameter. The background medium could be changed from lossless background to lossy. Furthermore, the transient RT theory could be applied to the broadband $\mathrm{THz}$ sensing and compared to time domain measurements. 
In this work, Mie size metal spheres were used in measurements, but various materials could be used for samples to obtain diffuse scattering; especially materials with $\mathrm{THz}$ spectral signatures, such as lactose, could be good material to detect its material properties. Also, a medium with high fractional volume of particles such as dense medium could be used to observe the relationship between fractional volume and diffuse scattering. Furthermore, samples containing particles with rough surface could be used in measurement and cross polarization measurements could be conducted. 


\section{References}

[1] S.P. Mickan and X.-C. Zhang. T-ray sensing and imaging. International Journal of High Speed Electronics and Systems, 13(2):601 - 676, 2003.

[2] Martin van Exter and Daniel R. Grischkowsky. Characterization of an optoelectronic terahertz beam system. IEEE Transactions on Microwave Theory and Techniques, 38(11):1684 - 1691, 1990.

[3] Philip C. Ashworth, Emma Pickwell-MacPherson, Elena Provenzano, Sarah E. Pinder, Anand D. Purushotham, Michael Pepper, and Vincent P. Wallace. Terahertz pulsed spectroscopy of freshly excised human breast cancer. Optics Express, 17(15):12444 - 12454, 2009.

[4] P. Knobloch, K. Schmalstieg, M. Koch, E. Rehberg, F. Vauti, and K. Donhuijsen. Thz imaging of histo-pathological samples. volume 4434, pages 239 $-245,2001$.

[5] M.C. Kemp, P.F. Taday, B.E. Cole, J.A. Cluff, A.J. Fitzgerald, and W.R. Tribe. Security applications of terahertz technology. volume 5070, pages 44 $-52,2003$. 
[6] John F. Federici, Brian Schulkin, Feng Huang, Dale Gary, Robert Barat, Filipe Oliveira, and David Zimdars. Thz imaging and sensing for security applications - explosives, weapons and drugs. Semiconductor Science and Technology, 20(7):S266 - S280, 2005.

[7] Thomas Kurner, Radoslaw Piesiewicz, Martin Koch, and Joerg Schoebel. Propagation models, measurements and simulations for wireless communication systems beyond 100 ghz. pages $108-111,2007$.

[8] Radoslaw Piesiewicz, Christian Jansen, Daniel Mittleman, Thomas KleineOstmann, Martin Koch, and Thomas Kurner. Scattering analysis for the modeling of thz communication systems. IEEE Transactions on Antennas and Propagation, 55(11 I):3002 - 3009, 2007.

[9] Yunqing Chen, Haibo Liu, Michel J. Fitch, Robert Osiander, James B. Spicer, Michael Shur, and X.-C. Zhang. Thz diffuse reflectance spectra of selected explosives and related compounds. volume 5790, pages $19-24$, 2005.

[10] Lisa M. Zurk, Brian Orlowski, Dale P. Winebrenner, Eric I. Thorsos, Megan Leahy-Hoppa, and Michael R. Hayden. Terahertz scattering from granular material. Journal of the Optical Society of America B: Optical Physics, 24(9):2238 - 2243, 2007. 
[11] M. Hassan Arbab, Antao Chen, Eric I. Thorsos, Dale P. Winebrenner, and Lisa M. Zurk. Effect of surface scattering on terahertz time domain spectroscopy of chemicals. volume 6893, 2008.

[12] S. Schecklman. Modeling terahertz scattering from random rough surfaces for reflection spectroscopy. Master's thesis, Portland State University, 2009.

[13] S.C. Henry, S. Schecklman, G.P. Kniffin, L.M. Zurk, and A. Chen. Measurement and modeling of rough surface effects on terahertz spectroscopy. volume 7601,2010 .

[14] Aparajita Bandyopadhyay, Amartya Sengupta, Robert B. Barat, Dale E. Gary, and John F. Federici. Grain size dependent scattering studies of common materials using thz time domain techniques. volume 6120, 2006.

[15] Jeremy Pearce and Daniel M. Mittleman. Propagation of single-cycle terahertz pulses in random media. Optics Letters, 26(24):2002 - 2004, 2001.

[16] K.J. Chau, S. Mujumdar, and A.Y. Elezzabi. Terahertz propagation in non-homogeneous strongly scattering media. volume 5727, pages 177 - 185 , 2005.

[17] X.-C. Zhang. Terahertz wave imaging: Horizons and hurdles. Physics in Medicine and Biology, 47(21):3667 - 3677, 2002. 
[18] Jeremy Pearce, Zhongping Jian, and Daniel M. Mittleman. Statistics of multiply scattered broadband terahertz pulses. Physical Review Letters, 91(4):043903/1 - 043903/4, 2003.

[19] Akira Ishimaru. Wave Propagation and Scattering in Random Media. IEEE Press-Oxford University Press, 1997.

[20] Leung Tsang and Jin Au Kong. Scattering of Electromagnetic Waves, Volume 3, Advanced Topics. John Wiley and Sons, 2001.

[21] S. Chandrasekhar. Radiative Transfer. Dover Publications, Inc., 1960.

[22] R.T. Shin and J.A. Kong. Radiative transfer theory for active remote sensing of a homogeneous layer containing spherical scatterers. Journal of Applied Physics, 52(6):4221 - 4230, 1981.

[23] L. Tsang and J.A. Kong. Radiative transfer theory for scattering by layered media. Journal of Applied Physics, 50(4):2465 - 2469, 1979.

[24] Leung Tsang and Jin Au Kong. Scattering of Electromagnetic Waves, Volume 1, Theories and Applications. John Wiley and Sons, 2000.

[25] Craig F. Bohren and Donald R. Huffman. Absorption and Scattering of Light by Small Par. Jo, 1983. 
[26] H.C. van de Hulst. Light Scattering by Small Particles. Dover Publications, Inc., 1957.

[27] Boheng Wen, Leung Tsang, Dale P. Winebrenner, and Akira Ishimaru. Dense medium radiative transfer theory: Comparison with experiment and application to microwave remote sensing and polarimetry. IEEE Transactions on Geoscience and Remote Sensing, 28(1):46 - 59, 1990.

[28] Leung Tsang, Chi-Te Chen, Alfred T. C. Chang, Jianjun Guo, and KungHau Ding. Dense media radiative transfer theory based on quasicrystalline approximation with applications to passive microwave remote sensing of snow. Radio Science, 35(3):731 - 749, 2000.

[29] Leung Tsang, Jin Pan, Ding Liang, Zhongxin Li, Donald W. Cline, and Yunhua Tan. Modeling active microwave remote sensing of snow using dense media radiative transfer (DMRT) theory with multiple-scattering effects. IEEE Transactions on Geoscience and Remote Sensing, 45(4):990 - 1004, 2007.

[30] L. Tsang and J.A. Kong. Multiple scattering of electromagnetic waves by random distributions of discrete scatterers with coherent potential and quantum mechanical formulism. Journal of Applied Physics, 51(7):3465 $3485,1980$. 
[31] L. Tsang and J.A. Kong. Effective propagation constants for coherent electromagnetic wave propagation in media embedded with dielectric scatters. Journal of Applied Physics, 53(11 pt 1):7162 - 7173, 1982.

[32] R. West, D. Gibbs, L. Tsang, and A.K. Fung. Comparison of optical scattering experiments and the quasi-crystalline approximation for dense media. Journal of the Optical Society of America A: Optics and Image Science, and Vision, 11(6):1854 - 1858, 1994.

[33] Leung Tsang and Jin Au Kong. Scattering of Electromagnetic Waves, Volume 2, Numerical Simulations. John Wiley and Sons, 2001.

[34] M. S. Wertheim. Exact solution of the percus-yevick integral equation for hard spheres. Phys. Rev. Lett., 10(8):321-323, Apr 1963.

[35] Hiroaki Yasuda and Iwao Hosako. Measurement of terahertz refractive index of metal with terahertz time-domain spectroscopy. Japanese Journal of Applied Physics, 47(3 PART 1):1632 - 1634, 2008.

[36] Urachada Ketprom, Yasuo Kuga, Sermsak Jaruwatanadilok, and Akira Ishimaru. Experimental and numerical analysis of polarized light through random distributed spherical particles. volume 4819, pages 35 - 45, 2002.

[37] Robert P. Cogdill, Steven M. Short, Ryanne Forcht, Zhenqi Shi, Yaochun Shen, Philip F. Taday, Carl A. Anderson, and James K. Drennen. An 
efficient method-development strategy for quantitative chemcal imaging using terahertz pulse spectroscopy. Journal of Pharmaceutical Innovation, September/October:63 - 75, 2006.

[38] E.R. Brown, J.E. Bjarnason, A.M. Fedor, and T.M. Korter. On the strong and narrow absorption signature in lactose at 0.53 thz. Applied Physics Letters, 90(6), 2007.

[39] Kohji Yamamoto, Mariko Yamaguchi, Fumiaki Miyamaru, Masahiko Tani, Masanori Hangyo, Takeshi Ikeda, Akira Matsushita, Kenji Koide, Michiaki Tatsuno, and Yukio Minami. Noninvasive inspection of c-4 explosive in mails by terahertz time-domain spectroscopy. Japanese Journal of Applied Physics, Part 2: Letters, 43(3 B):414-417 -, 2004.

[40] Rudolf Lap Tung Cheung and Akira Ishimaru. Transmission, backscattering, and depolarization of waves in randomly distributed spherical particles. Applied Optics, 21(20):3792 - 3798, 1982.

[41] Z. Sekera. Scattering matrices and reciprocity relationships for various representations of the state of polarization. Journal of the Optical Society of America B: Optical Physics, 56(12):1732-1740, December 1966. 


\section{Appendix A}

\section{Phase Matrix}

1) Phase Matrix For Independent Scattering

The phase matrix for independent scattering is expressed in terms of the scattering amplitudes:

$\overline{\bar{P}}=n_{o}\left[\begin{array}{cccc}\left|f_{v v}\right|^{2} & \left|f_{v h}\right|^{2} & \operatorname{Re}\left(f_{v v} f_{v h}^{*}\right) & -\operatorname{Im}\left(f_{v v} f_{v h}^{*}\right) \\ \left|f_{h v}\right|^{2} & \left|f_{h h}\right|^{2} & \operatorname{Re}\left(f_{h v} f_{h h}^{*}\right) & -\operatorname{Im}\left(f_{h v} f_{h h}^{*}\right) \\ 2 \operatorname{Re}\left(f_{v v} f_{h v}^{*}\right) & 2 \operatorname{Re}\left(f_{v h} f_{h h}^{*}\right) & \operatorname{Re}\left(f_{v v} f_{h h}^{*}+f_{v h} f_{h v}^{*}\right) & -\operatorname{Im}\left(f_{v v} f_{h h}^{*}-f_{v h} f_{h v}^{*}\right) \\ 2 \operatorname{Im}\left(f_{v v} f_{h v}^{*}\right) & 2 \operatorname{Im}\left(f_{v h} f_{h h}^{*}\right) & \operatorname{Im}\left(f_{v v} f_{h h}^{*}+f_{v h} f_{h v}^{*}\right) & \operatorname{Re}\left(f_{v v} f_{h h}^{*}-f_{v h} f_{h v}^{*}\right)\end{array}\right]$,

where $n_{o}$ is the number of particles per unit volume. The components of the above matrix $f_{a b}$ obtained from Cheung [40] represent the scattering amplitude function for a single spherical particle from Lorentz-Mie theory, including the rotation of the coordinate system to express it conveniently in a general coordinate system for multiple scattering $[40,41]$.

The scattering amplitude function including the rotation of the system is as 
follows:

$$
\begin{aligned}
& f_{v v}=(l, l) T_{1}+(r, r) T_{2}, \\
& f_{v h}=-(r, l) T_{1}+(l, r) T_{2}, \\
& f_{h v}=-(l, r) T_{1}+(r, l) T_{2}, \\
& f_{h h}=(r, r) T_{1}+(l, l) T_{2}, \\
& (l, l)=\left[\left(1-\mu^{2}\right)\left(1-\mu^{\prime 2}\right)\right]^{1 / 2}+\mu \mu^{\prime} \cos \left(\phi^{\prime}-\phi\right), \\
& (l, r)=-\mu^{\prime} \sin \left(\phi^{\prime}-\phi\right), \\
& (r, l)=\mu \sin \left(\phi^{\prime}-\phi\right), \\
& (r, r)=\cos \left(\phi^{\prime}-\phi\right), \\
& T_{1}(\chi)=\frac{A_{r r}(\chi)-\chi A_{l l}(\chi)}{1-\chi^{2}}, \\
& T_{2}(\chi)=\frac{A_{l l}(\chi)-\chi A_{r r}(\chi)}{1-\chi^{2}}, \\
& \chi=\cos \Theta=\left[\left(1-\mu^{2}\right)\left(1-\mu^{\prime 2}\right)\right]^{1 / 2} \cos \left(\phi^{\prime}-\phi\right)+\mu \mu^{\prime}, \\
& A_{l l}=S_{2} / j k, \\
& A_{r r}=S_{1} / j k,
\end{aligned}
$$

where $S_{1}$ and $S_{2}$ are the scattering amplitudes representing the relationship between the incident field and the scattered field used by van de Hulst [26]; 


$$
\begin{aligned}
& S_{1}(\cos \Theta)=\sum_{n=1}^{\infty} \frac{2 n+1}{n(n+1)}\left(a_{n} \pi_{n}+b_{n} \tau_{n}\right), \\
& S_{2}(\cos \Theta)=\sum_{n=1}^{\infty} \frac{2 n+1}{n(n+1)}\left(a_{n} \tau_{n}+b_{n} \pi_{n}\right),
\end{aligned}
$$

where $a_{n}$ and $b_{n}$ are the scattering coefficients from Mie theory.

\section{2) Phase Matrix For DMRT}

The phase matrix for dense media radiative transfer theory is derived from [29]. The parameters $A_{l l}=S_{2} / j k$ and $A_{r r}=S_{1} / j k$ are replaced with $A_{l l}=f_{22}$, and $A_{r r}=f_{11}$ in Equation (2), where $f_{11}$ and $f_{22}$ are not the scattering amplitudes, but are defined as follows [29]:

$$
\begin{aligned}
f_{11}(\Theta) & =-\frac{i}{1-R} \sqrt{\frac{1}{k K_{r}}} \sum_{n=1}^{N_{\max }} \frac{2 n+1}{n(n+1)} \\
& \times\left[T_{n}^{(M)} X_{n}^{(M)} \tau_{n}(\cos \Theta)+T_{n}^{(N)} X_{n}^{(N)} \pi_{n}(\cos \Theta)\right]
\end{aligned}
$$

$$
\begin{aligned}
f_{22}(\Theta) & =-\frac{i}{1-R} \sqrt{\frac{1}{k K_{r}}} \sum_{n=1}^{N_{\max }} \frac{2 n+1}{n(n+1)} \\
& \times\left[T_{n}^{(M)} X_{n}^{(M)} \pi_{n}(\cos \Theta)+T_{n}^{(N)} X_{n}^{(N)} \tau_{n}(\cos \Theta)\right]
\end{aligned}
$$


The QCA/DMRT phase matrix is

$$
\overline{\bar{P}}=q(\Theta)\left[\begin{array}{cccc}
\left|f_{v v}\right|^{2} & \left|f_{v h}\right|^{2} & \operatorname{Re}\left(f_{v v} f_{v h}^{*}\right) & -\operatorname{Im}\left(f_{v v} f_{v h}^{*}\right) \\
\left|f_{h v}\right|^{2} & \left|f_{h h}\right|^{2} & \operatorname{Re}\left(f_{h v} f_{h h}^{*}\right) & -\operatorname{Im}\left(f_{h v} f_{h h}^{*}\right) \\
2 \operatorname{Re}\left(f_{v v} f_{h v}^{*}\right) & 2 \operatorname{Re}\left(f_{v h} f_{h h}^{*}\right) & \operatorname{Re}\left(f_{v v} f_{h h}^{*}+f_{v h} f_{h v}^{*}\right) & -\operatorname{Im}\left(f_{v v} f_{h h}^{*}-f_{v h} f_{h v}^{*}\right) \\
2 \operatorname{Im}\left(f_{v v} f_{h v}^{*}\right) & 2 \operatorname{Im}\left(f_{v h} f_{h h}^{*}\right) & \operatorname{Im}\left(f_{v v} f_{h h}^{*}+f_{v h} f_{h v}^{*}\right) & \operatorname{Re}\left(f_{v v} f_{h h}^{*}-f_{v h} f_{h v}^{*}\right)
\end{array}\right],
$$

where $q(\Theta)=n_{o} S(\Theta)$ and $S(\Theta)$ is structure factor, defined as $S(\Theta)=1+$ $n_{o}(2 \pi)^{3} H(\Theta)$. Here, $H(\Theta)$ is

$$
H(\Theta)=\frac{1}{(2 \pi)^{3}} \int_{-\infty}^{\infty} d \bar{r} e^{-i \bar{p} \cdot \bar{r}}(g(\bar{r})-1)
$$

where $p=\left[\operatorname{Re}(K)^{2}+k^{2}-2 k \operatorname{Re}(K) \cos \Theta\right]^{1 / 2}$, and $g(r)$ is Percus-Yevick pair distribution function for hard spheres. 


\section{Appendix B}

\section{Reflectivity Matrix and Transmissivity Matrix}

The reflectivity matrix and the transmissivity matrix are identical to Equations (7.2.93) and (7.2.95) from [24].

The reflectivity matrix is given by [24]

$$
\overline{\bar{R}}\left(\theta_{i}\right)=\left[\begin{array}{cccc}
r_{v}\left(\theta_{i}\right) & 0 & 0 & 0 \\
0 & r_{h}\left(\theta_{i}\right) & 0 & 0 \\
0 & 0 & \operatorname{Re}\left[R_{v}\left(\theta_{i}\right) R_{h}^{\star}\left(\theta_{i}\right)\right] & -\operatorname{Im}\left[R_{v}\left(\theta_{i}\right) R_{h}^{\star}\left(\theta_{i}\right)\right] \\
0 & 0 & \operatorname{Im}\left[R_{v}\left(\theta_{i}\right) R_{h}^{\star}\left(\theta_{i}\right)\right] & \operatorname{Re}\left[R_{v}\left(\theta_{i}\right) R_{h}^{*}\left(\theta_{i}\right)\right]
\end{array}\right]
$$

where $\theta_{i}$ is the incident angle in medium 1 at the interface with medium 2 and the subscripts $v$ and $h$ mean the vertical polarization and the horizontal polarization, respectively. 
The transmissivity matrix is

$$
\overline{\bar{T}}\left(\theta_{i}\right)=\frac{\epsilon_{2}}{\epsilon_{1}}\left[\begin{array}{cccc}
t_{v}\left(\theta_{i}\right) & 0 & 0 & 0 \\
0 & t_{h}\left(\theta_{i}\right) & 0 & 0 \\
0 & 0 & c\left(\theta_{i}\right) \operatorname{Re}\left[T_{v}\left(\theta_{i}\right) T_{h}^{*}\left(\theta_{i}\right)\right] & -c\left(\theta_{i}\right) \operatorname{Im}\left[T_{v}\left(\theta_{i}\right) T_{h}^{*}\left(\theta_{i}\right)\right] \\
0 & 0 & c\left(\theta_{i}\right) \operatorname{Im}\left[T_{v}\left(\theta_{i}\right) T_{h}^{*}\left(\theta_{i}\right)\right] & c\left(\theta_{i}\right) \operatorname{Re}\left[T_{v}\left(\theta_{i}\right) T_{h}^{*}\left(\theta_{i}\right)\right]
\end{array}\right],
$$

where $c=\frac{\cos \theta_{t}}{\cos \theta_{i}}$ and the angle $\theta_{t}$ is the transmitted angle of $\theta_{i}$ from Snell's law. 


\section{Appendix C}

\section{Numerical Solution for Diffuse Intensity}

Shin [22] and Wen [27] solved the vector radiative transfer equations for independent Rayleigh scattering and dense medium Rayleigh scattering, respectively. The procedures for solving VRTE for independent Mie scattering numerically follows Shin and Wen's solutions and the notation will be similar.

The first step in solving the radiative transfer equation for diffuse intensity is to apply a Fourier series expansion in the azimuthal direction to the specific intensity and the phase matrix, which results in $\theta$ and $z$ dependence and independence of $\phi$ in the RT equation.

The Fourier series expansions of the specific intensity and the phase matrix are

$$
\bar{I}(\theta, \phi, z)=\sum_{m=0}^{\infty}\left[\bar{I}^{m c}(\theta, z) \cos m\left(\phi-\phi_{i}\right)+\bar{I}^{m s}(\theta, z) \sin m\left(\phi-\phi_{i}\right)\right]
$$


and

$$
\overline{\bar{P}}\left(\theta, \phi ; \theta^{\prime}, \phi^{\prime}\right)=\sum_{m=0}^{\infty}\left[\overline{\bar{P}}^{m c}\left(\theta, \theta^{\prime}\right) \cos m\left(\phi-\phi^{\prime}\right)+\overline{\bar{P}}^{m s}\left(\theta, \theta^{\prime}\right) \sin m\left(\phi-\phi^{\prime}\right)\right]
$$

where $\phi_{i}$ is the incident angle in the $\phi$ direction, and the superscript $m c$ and $m s$ are the $m_{t h}$ order harmonic of the Fourier series expansion with cosine and sine dependence in the $\phi$ direction, respectively.

These equations are substituted into the RT equation, and then integration is carried out with respect to $\phi^{\prime}$ using the trigonometric addition formulas and the orthogonality of trigonometric functions. Next, after collecting and rearranging the sine and cosine terms, we have

$$
\begin{aligned}
\cos \theta \frac{\mathrm{d}}{\mathrm{d} z} \bar{I}_{d}^{m \alpha}(\theta, z) & =-\kappa_{e} \bar{I}_{d}^{m \alpha}(\theta, z)+\left(1+\delta_{m}\right) \pi \int_{o}^{\pi} \mathrm{d} \theta^{\prime} \sin \theta^{\prime} \overline{\bar{P}}^{m \alpha}\left(\theta ; \theta^{\prime}\right) \bar{I}_{d}^{m \alpha}\left(\theta^{\prime}, z\right) \\
& +\overline{\bar{P}}^{m \alpha}\left(\theta ; \theta_{1 i}\right) \bar{J}_{1}^{m \alpha}\left(\theta_{1 i}\right) \exp \left[-\kappa_{e}(z+2 d) \sec \theta_{1 i}\right] \\
& +\overline{\bar{P}}^{m \alpha}\left(\theta ; \pi-\theta_{1 i}\right) \bar{J}_{2}^{m \alpha}\left(\theta_{1 i}\right) \exp \left(\kappa_{e} z \sec \theta_{1 i}\right)
\end{aligned}
$$

where $\alpha$ is an even or odd term, which is rearranged sine term and cosine term. 
The even and odd terms of specific intensity are defined as

$$
\bar{I}_{d}^{m \alpha}(\theta, z)=\left[\begin{array}{c}
I_{v}^{m c}(\theta, z) \\
I_{h}^{m c}(\theta, z) \\
U^{m s}(\theta, z) \\
V^{m s}(\theta, z)
\end{array}\right]_{\alpha=e}, \bar{I}_{d}^{m \alpha}(\theta, z)=\left[\begin{array}{c}
I_{v}^{m s}(\theta, z) \\
I_{h}^{m s}(\theta, z) \\
U^{m c}(\theta, z) \\
V^{m c}(\theta, z)
\end{array}\right]_{\alpha=o} .
$$

The even and odd terms of the phase matrix are given as

$$
\overline{\bar{P}}^{m \alpha}\left(\theta ; \theta^{\prime}\right)=\left[\begin{array}{cccc}
P_{11}^{m c}\left(\theta ; \theta^{\prime}\right) & P_{12}^{m c}\left(\theta ; \theta^{\prime}\right) & -P_{13}^{m s}\left(\theta ; \theta^{\prime}\right) & -P_{14}^{m s}\left(\theta ; \theta^{\prime}\right) \\
P_{21}^{m c}\left(\theta ; \theta^{\prime}\right) & P_{22}^{m c}\left(\theta ; \theta^{\prime}\right) & -P_{23}^{m s}\left(\theta ; \theta^{\prime}\right) & -P_{24}^{m s}\left(\theta ; \theta^{\prime}\right) \\
P_{31}^{m s}\left(\theta ; \theta^{\prime}\right) & P_{32}^{m s}\left(\theta ; \theta^{\prime}\right) & P_{33}^{m c}\left(\theta ; \theta^{\prime}\right) & P_{34}^{m c}\left(\theta ; \theta^{\prime}\right) \\
P_{41}^{m s}\left(\theta ; \theta^{\prime}\right) & P_{42}^{m s}\left(\theta ; \theta^{\prime}\right) & P_{43}^{m c}\left(\theta ; \theta^{\prime}\right) & P_{44}^{m c}\left(\theta ; \theta^{\prime}\right)
\end{array}\right]_{\alpha=e}
$$

and

$$
\overline{\bar{P}}^{m \alpha}\left(\theta ; \theta^{\prime}\right)=\left[\begin{array}{cccc}
P_{11}^{m c}\left(\theta ; \theta^{\prime}\right) & P_{12}^{m c}\left(\theta ; \theta^{\prime}\right) & P_{13}^{m s}\left(\theta ; \theta^{\prime}\right) & P_{14}^{m s}\left(\theta ; \theta^{\prime}\right) \\
P_{21}^{m c}\left(\theta ; \theta^{\prime}\right) & P_{22}^{m c}\left(\theta ; \theta^{\prime}\right) & P_{23}^{m s}\left(\theta ; \theta^{\prime}\right) & P_{24}^{m s}\left(\theta ; \theta^{\prime}\right) \\
-P_{31}^{m s}\left(\theta ; \theta^{\prime}\right) & -P_{32}^{m s}\left(\theta ; \theta^{\prime}\right) & P_{33}^{m c}\left(\theta ; \theta^{\prime}\right) & P_{34}^{m c}\left(\theta ; \theta^{\prime}\right) \\
-P_{41}^{m s}\left(\theta ; \theta^{\prime}\right) & -P_{42}^{m s}\left(\theta ; \theta^{\prime}\right) & P_{43}^{m c}\left(\theta ; \theta^{\prime}\right) & P_{44}^{m c}\left(\theta ; \theta^{\prime}\right)
\end{array}\right]_{\alpha=o}
$$


$\bar{J}_{1}^{m \alpha}\left(\theta, \theta_{1 i}\right)$ and $\bar{J}_{2}^{m \alpha}\left(\theta, \theta_{1 i}\right)$ are

$$
\begin{aligned}
& \bar{J}_{1}^{m \alpha}\left(\theta, \theta_{1 i}\right)=\frac{n_{0}^{2} \cos \theta_{0 i}}{n_{1}^{2} \cos \theta_{1 i}} \overline{\bar{F}}\left(\theta_{1 i}\right) \overline{\bar{R}}_{12}\left(\theta_{1 i}\right) \overline{\bar{T}}_{01}\left(\theta_{0 i}\right) \bar{I}_{1 i}^{m \alpha}\left(\pi-\theta_{1}\right), \\
& \bar{J}_{2}^{m \alpha}\left(\theta, \theta_{1 i}\right)=\frac{n_{0}^{2} \cos \theta_{0 i}}{n_{1}^{2} \cos \theta_{1 i}} \overline{\bar{F}}\left(\theta_{1 i}\right) \overline{\bar{T}}_{01}\left(\theta_{0 i}\right) \bar{I}_{1 i}^{m \alpha}\left(\pi-\theta_{1}\right) .
\end{aligned}
$$


The next step is to use the Gaussian quadrature method to discretize the variable $\theta$ in the integral term of Equation (3):

$$
\begin{aligned}
\mu_{k} \frac{d}{d z} \bar{I}_{d}^{m \alpha}\left(\mu_{k}, z\right) & =-\kappa_{e} \bar{I}_{d}^{m \alpha}\left(\mu_{k}, z\right)+\left(1+\delta_{m}\right) \pi \sum_{j=-N}^{N} a_{j} \overline{\bar{P}}^{m \alpha}\left(\mu_{k}, \mu_{j}\right) \bar{I}_{d}^{m \alpha}\left(\mu_{j}, z\right) \\
& +\overline{\bar{P}}^{m \alpha}\left(\mu_{k} ; \mu_{1 i}\right) \bar{J}_{1}^{m \alpha}\left(\mu_{1 i}\right) \exp \left[-\kappa_{e}(z+2 d) / \mu_{1 i}\right] \\
& +\overline{\bar{P}}^{m \alpha}\left(\mu_{k} ;-\mu_{1 i}\right) \bar{J}_{2}^{m \alpha}\left(\mu_{1 i}\right) \exp \left(\kappa_{e} z / \mu_{1 i}\right) .
\end{aligned}
$$

The discretizing angles $\mu_{k}=\cos \theta_{k}$ are the zeros of the Legendre polynomial $P_{2 N}(\mu), k=-N,-N+1, \cdots,-1,1, \cdots, N-1, N$ and the weight $a_{j}$ is referred to as the Christoffel number.

We can rewrite Equation (8) in compact matrix form such that

$$
\frac{\mathrm{d}}{\mathrm{d} z} \mathbf{I}_{d}^{m \alpha}(z)+\mathbf{S}^{m \alpha} \mathbf{I}_{d}^{m \alpha}(z)=\mathbf{B}_{1}^{m \alpha} \exp \left[-\kappa_{e}(z+2 d) / \mu_{1 i}\right]+\mathbf{B}_{2}^{m \alpha} \exp \left(\kappa_{e} z / \mu_{1 i}\right),
$$

where $\mathbf{I}_{d}^{m \alpha}$ is a $8 N \times 1$ vector, $\mathbf{S}^{m \alpha}$ is a $8 N \times 8 N$ matrix, and $\mathbf{B}_{1}^{m \alpha}$ and $\mathbf{B}_{2}^{m \alpha}$ are each $8 N \times 1$ vectors, 


$$
\left.\begin{array}{c}
\mathbf{I}_{d}^{m \alpha}=\left[\begin{array}{c}
\bar{I}_{d}^{m \alpha}\left(\mu_{-N}, z\right) \\
\vdots \\
\bar{I}_{d}^{m \alpha}\left(\mu_{-1}, z\right) \\
\bar{I}_{d}^{m \alpha}\left(\mu_{1}, z\right) \\
\vdots \\
\mathbf{S}^{m \alpha}
\end{array}\right], \text { and } \\
\bar{I}_{d}^{m \alpha}\left(\mu_{N}, z\right)
\end{array}\right]
$$


where diag means a diagonal matrix, and

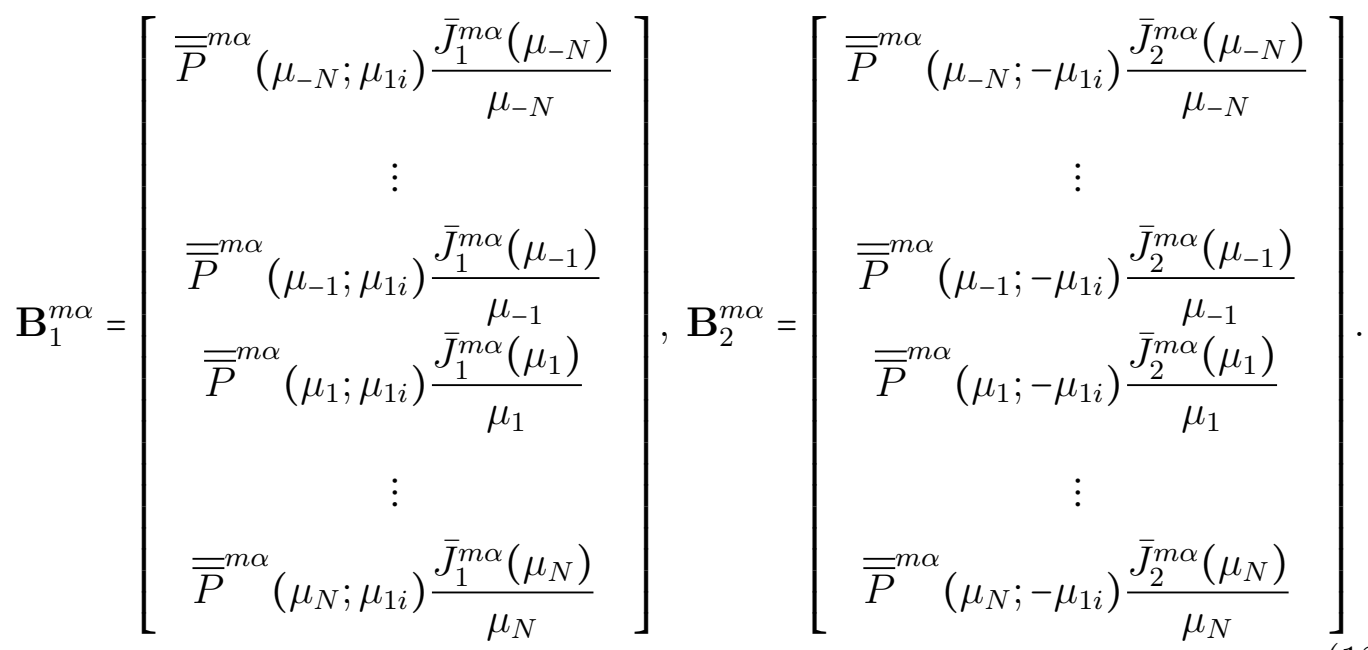

Equation (9), which is a linear first order differential equation in matrix form, has particular solutions and homogeneous solutions. The particular solution has the following form

$$
\mathbf{I}_{p}^{m \alpha}=\mathbf{a}_{1}^{m \alpha} \exp \left[-\kappa_{e}(z+2 d) / \mu_{1 i}\right]+\mathbf{a}_{2}^{m \alpha} \exp \left(\kappa_{e} z / \mu_{1 i}\right)
$$

Substituting the particular solution into Equation (9), we can obtain coefficients

$$
\begin{gathered}
\mathbf{a}_{1}^{m \alpha}=\left[\mathbf{S}^{m \alpha}-\frac{\kappa_{e}}{\mu_{1 i}} \mathbf{U}\right]^{-1} \mathbf{B}_{1}^{m \alpha}, \text { and } \\
\mathbf{a}_{2}^{m \alpha}=\left[\mathbf{S}^{m \alpha}+\frac{\kappa_{e}}{\mu_{1 i}} \mathbf{U}\right]^{-1} \mathbf{B}_{2}^{m \alpha},
\end{gathered}
$$


where $\mathbf{U}$ is an $8 N \times 8 N$ identity matrix.

The homogeneous equation and its general solution are

$$
\frac{d}{d z} \mathbf{I}_{h}^{m \alpha}+\mathbf{S}^{m \alpha} \mathbf{I}_{h}^{m \alpha}=0
$$

$$
\mathbf{I}_{h}^{m \alpha}=\mathbf{b}^{m \alpha} e^{-\lambda z} .
$$

Substituting the general solution into the homogeneous equation, we have a matrix eigenvalue problem with eigenvalues $\lambda$ and eigenvector $\mathbf{b}$. The homogeneous solution can be obtained using a linear combination of all eigenvectors with coefficients, $C_{n}$.

The homogeneous solution can be explicitly written as

$$
\mathbf{I}_{h}^{m \alpha}=\sum_{n=1}^{8 N} C_{n}^{m \alpha} \mathbf{b}_{n}^{m \alpha} \exp \left(-\lambda_{n}^{m \alpha} z\right)
$$

Thus the summation of Eq. (13) and Eq. (18) is the diffuse intensity,

$$
\mathbf{I}_{d}^{m \alpha}=\mathbf{I}_{p}^{m \alpha}(z)+\sum_{n=1}^{8 N} C_{n}^{m \alpha} \mathbf{b}_{n}^{m \alpha} \exp \left(-\lambda_{n}^{m \alpha} z\right) .
$$

The final step is to calculate the unknown coefficients $C_{n}$ using the boundary conditions for the diffuse intensity matrix such that 


$$
\begin{gathered}
\mathbf{I}_{-}^{m \alpha}(z=0) \quad=\quad \mathbf{R}_{10} \mathbf{I}_{+}^{m \alpha}(z=0) \\
\text { and } \\
\mathbf{I}_{+}^{m \alpha}(z=-d) \quad=\quad \mathbf{R}_{12} \mathbf{I}_{-}^{m \alpha}(z=-d),
\end{gathered}
$$

where $\mathbf{I}_{+}^{m \alpha}$ represents the upward going intensities and $\mathbf{I}_{-}^{m \alpha}$ represents the downward going intensities.

The reflectivitity matrix $\mathbf{R}$ is

$$
\mathbf{R}=\left[\begin{array}{ccc}
\overline{\bar{R}}\left(\theta_{N}\right) & \cdots & 0 \\
\vdots & \vdots & \vdots \\
0 & \cdots & \overline{\bar{R}}\left(\theta_{1}\right)
\end{array}\right]
$$

As a result, we have two equations;

$$
\left(\mathbf{a}_{1,-}^{m \alpha}-\mathbf{R}_{10} \mathbf{a}_{1,+}^{m \alpha}\right) e^{-\kappa_{e} 2 d / \mu_{1 i}}+\left(\mathbf{a}_{2,-}^{m \alpha}-\mathbf{R}_{10} \mathbf{a}_{2,+}^{m \alpha}\right)+\left(\widehat{\mathbf{b}}_{-}^{m \alpha}-\mathbf{R}_{10} \widehat{\mathbf{b}}_{+}^{m \alpha}\right) \mathbf{C}^{m \alpha}=0
$$

and 


$$
\left(\mathbf{a}_{1,+}^{m \alpha}-\mathbf{R}_{12} \mathbf{a}_{1,-}^{m \alpha}\right) e^{-\kappa_{e} d / \mu_{1 i}}+\left(\mathbf{a}_{2,+}^{m \alpha}-\mathbf{R}_{12} \mathbf{a}_{2,-}^{m \alpha}\right) e^{-\kappa_{e} d / \mu_{1 i}}+\left(\widehat{\mathbf{b}}_{+}^{m \alpha}-\mathbf{R}_{12} \widehat{\mathbf{b}}_{-}^{m \alpha}\right) \mathbf{L}^{m \alpha} \mathbf{C}^{m \alpha}=0
$$

where $\mathbf{L}^{m \alpha}$ is an $8 N \times 8 N$ matrix,

$$
\mathbf{L}^{m \alpha}=\operatorname{diag}\left[\exp \left(\lambda_{1} d\right), \exp \left(\lambda_{2} d\right), \cdots, \exp \left(\lambda_{8 N} d\right)\right]
$$

and $\widehat{\mathbf{b}}^{m \alpha}$ is an $8 N \times 8 N$ matrix,

$$
\widehat{\mathbf{b}}^{m \alpha}=\left[\begin{array}{llll}
\mathbf{b}_{1}^{m \alpha}, & \cdots, & \mathbf{b}_{8 N}^{m \alpha}
\end{array}\right]=\left[\begin{array}{ccc}
\bar{b}_{-N, 1}^{m \alpha}, & \cdots, & \bar{b}_{-N, 8 N}^{m \alpha} \\
\vdots, & \vdots & \vdots \\
\bar{b}_{N, 1}^{m \alpha} & \cdots & \bar{b}_{N, 8 N}^{m \alpha}
\end{array}\right] .
$$

Combining Equations (22) and (23), we have a single matrix equation

$$
\mathbf{D}^{m \alpha}+\mathbf{M}^{m \alpha} \mathbf{C}^{m \alpha}=0
$$

where $\mathbf{D}^{m \alpha}$ is an $8 N \times 1$ vector,

$$
\mathbf{D}^{m \alpha}=\left[\begin{array}{c}
\left(\mathbf{a}_{1,-}^{m \alpha}-\mathbf{R}_{10} \mathbf{a}_{1,+}^{m \alpha}\right) \exp \left(-\kappa_{e} 2 d / \mu_{1 i}\right)+\left(\mathbf{a}_{2,-}^{m \alpha}-\mathbf{R}_{10} \mathbf{a}_{2,+}^{m \alpha}\right) \\
\left(\mathbf{a}_{1,+}^{m \alpha}-\mathbf{R}_{12} \mathbf{a}_{1,-}^{m \alpha}\right) \exp \left[-\kappa_{e} d / \mu_{1 i}\right]+\left(\mathbf{a}_{2,+}^{m \alpha}-\mathbf{R}_{12} \mathbf{a}_{2,-}^{m \alpha}\right) \exp \left(-\kappa_{e} d / \mu_{1 i}\right)
\end{array}\right]
$$


and $\mathbf{M}^{m \alpha}$ is an $8 N \times 8 N$ matrix,

$$
\mathbf{M}^{m \alpha}=\left[\begin{array}{c}
\widehat{\mathbf{b}}_{-}^{m \alpha}-\mathbf{R}_{10} \widehat{\mathbf{b}}_{+}^{m \alpha} \\
\left(\widehat{\mathbf{b}}_{+}^{m \alpha}-\mathbf{R}_{12} \widehat{\mathbf{b}}_{-}^{m \alpha}\right) \mathbf{L}^{m \alpha}
\end{array}\right]
$$

The coefficients $\mathbf{C}^{m \alpha}=\left(\mathbf{M}^{m \alpha}\right)^{-1} \mathbf{D}^{m \alpha}$ are substituted into Equation (19). With the even and odd terms of diffuse intensity in Equation (19), and after rearranging the cosine and sine terms of the diffuse intensity, we can obtain the final solution of diffuse intensity with $\phi$ dependence by substituting the cosine and sine terms into Equation (1). 